\title{
Aerofoil dipole noise due to flow separation and stall at a low Reynolds number
}

\author{
Jacob M. Turner, Jae Wook Kim \\ Institute of Sound and Vibration Research, University of Southampton, \\ Southampton, SO17 1BJ, UK
}

\begin{abstract}
Aerofoil self-noise produced by flow separation and stall is relatively little understood regarding the underlying generation mechanisms. The focus of this work is to provide an improved level of understanding particularly with regard to the dipole noise sources utilising a high-fidelity direct numerical simulation. A NACA0012 aerofoil is considered under three different flow conditions at a Reynolds number $R e_{\infty}=50,000$ and a Mach number $M_{\infty}=0.4$. These include: a pre-stall condition with a laminar separation bubble $\left(\alpha=5^{\circ}\right)$, near-stall $\left(\alpha=10^{\circ}\right)$, and fully stalled $\left(\alpha=15^{\circ}\right)$. The noise radiation in the far-field is significantly increased at low frequencies for the full-stall case for all observer directions which is consistent with previous experimental observations. The dominant source regions for each configuration are identified for low, medium and high frequencies, separately. A number of key findings are made concerning the source characteristics in full-stall case which differ considerably from the lower angle of attack cases. It is found that the location of the dominant sources changes more significantly with frequency for the full-stall case. Additionally, for medium to high frequencies the maximum acoustic source amplitude is weaker for the full-stall case, despite comparable levels observed in the far-field. This seemingly contradictory observation highlights the importance of phase variations in the wall pressure fluctuations. For the frequencies considered in this paper it is shown that the full-stall case usually produces a relatively more in-phase source distribution, resulting in a more efficient radiation despite the lower amplitude levels. The important flow structures which are responsible for the dipole sources are also identified through analysis of the pressure field at isolated frequencies. It is found that for low frequencies coherent structures in the shear layer are responsible for the scattering of the wall pressure fluctuations at the TE, which agrees with previous findings in the literature. However, at medium and high frequencies the shear layer structures are found to be relatively weak in the proximity of the TE. This indicates that the noise may be generated through other means, for example scattering of fluctuating pressure induced by vortices shed from the TE.
\end{abstract}

Keywords: Aerofoil stall; Flow separation; Aeroacoustics

\section{Introduction}

Noise from aerofoil flow separation and stall is a relatively under-explained source of aerodynamic sound which is significant for many turbomachinery applications where unsteady inflow conditions may are encountered. One such example is wind turbine operation, where stalled conditions may be encountered due 5 to either wind shearing, or wake-blade interaction [1]. Historically, the majority of aerofoil self-noise research has focused on understanding the mechanisms of noise generation from scattering of a turbulent boundary layer at an aerofoil trailing edge (TBL-TE). Despite this, it has been suggested that noise from flow separation could reasonably exceed low angle TBL-TE by more than $10 \mathrm{~dB}[2$. Presently, there is a lack of

\footnotetext{
* Corresponding author

Email address: J.M.Turner@soton.ac.uk (Jacob M. Turner)
} 
reported information regarding the significance of stall noise, particular concerning high fidelity numerical 10 data, which may be useful for identifying the underlying mechanisms.

The most prominent observation in the literature is that the stall is characterised by a high level of low frequency sound [2]. More specifically, in the light stall regime increased low frequency broadband levels are observed, while in deep stall low frequency tones are also exhibited 5. Regarding the influence of aerofoil geometry, Laratro et al. [7] demonstrated comparable sound signatures for NACA0012 and NACA0021 aerofoils while in deep stall. However, differences were observed at stall onset, which were attributed to the severity of lift reduction encountered at the stall angle. Currently there is a lack of aerofoil stall noise prediction models, with most relying on prior knowledge of the flow. Perhaps the most well known is the semi-empirical model developed by Brooks et al. 4, which has been successfully used to predict broadband $1 / 3$ octave spectra in the stall regime, but is incapable of replicating the tonal noise observed at low frequencies. In an attempt to rectify this, Moreau et al. [5] have developed a low frequency model based on Curle's analogy and compact source assumptions which was shown to agree well with experimental data. A similar approach was also taken by Bertagnolio et al. [6] who identified a scaling law for different aerofoil geometries and a range of Reynolds numbers. These models rely on experimental measurements of wall pressure fluctuations and correlation length. In order to establish analytical prediction models requiring only on initial conditions it is apparent that better understanding of the noise generation mechanisms will be required.

At present there is no generally agreed consensus for the stall noise generation mechanisms (in part due to the limited amount of the design space which has been explored). However, it is clear that stall noise operates under different mechanisms to TBL-TE noise. An early example of this is the experimental study 30 by Paterson et al. 3 who compared the two-point correlation between the wall pressure fluctuations and the radiated sound field. The eddy convection velocity was calculated by considering the cross-correlation zero-crossings between microphones on the surface and in the far-field. This resulted in an unreasonably large prediction for the final $30 \%$ of the chord, which indicated that the radiated noise may be generated further upstream from the TE. Similarly, Mayer et al. 9] have demonstrated an increase in low frequency 35 energy and coherence along the full aerofoil chord as the angle of attack is increased into the stall regime. These results also indicate how the same noise reduction techniques for example TE serrations may be less effective for stall noise than TBL-TE where the sources are highly concentrated at the TE. A recent study by Lacagnina et al. [8] calculated the coherence between hot-wire velocity measurements in the separated shear layer and the pressure at the aerofoil TE. Modal analysis of particle image velocimetry data was 40 carried out at frequencies with high coherence in order to identify the flow structures responsible for the TE pressure changes. Three possible mechanisms for the noise generation were suggested for an aerofoil near stall conditions. These were: shear layer instabilities, shear layer flapping, and shedding of coherent structures.

The literature highlights a requirement for high fidelity numerical simulations in order to better un45 derstand the noise generation mechanisms in stall. In particular a simulation approach can be useful for determining the source distribution over the entire aerofoil surface. Direct numerical simulations (DNS) of a NACA0012 aerofoil are considered in the current paper at a low Reynolds number $R e_{\infty}=50,000$ and moderate Mach number $M_{\infty}=0.4$. Under these conditions the aerofoil encounters LE stall as the angle of attack is increased. Three specific angles are considered in the current work: $\alpha=5^{\circ}$ where a laminar 50 separation bubble is observed around the aerofoil mid-chord; $\alpha=10^{\circ}$ which is a near stall configuration, with the bubble located near the LE; and $\alpha=15^{\circ}$, a fully stalled condition. The paper makes a number of new findings concerning the distribution of the acoustic sources over the wall in stall. The significance of source amplitude as well as phase variation is highlighted for the three angles of attack for low, medium and high frequency ranges. Additionally, frequency filtered pressure field data is utilised to identify the key flow structures for each case in the current flow configuration.

The remainder of the paper is organized as follows: Section 2 provides an overview of the methodology. This includes the numerical schemes, boundary conditions, far-field sound extrapolation technique and details of the computational mesh. The DNS results are provided in section 3 including aerodynamic data and flow visualisation. In section 3.1 the far-field noise and directivity patterns are compared for the three so angles of attack. In section 3.2 the dipole sources are identified and discussed in detail by considering the 
wall pressure data over the full aerofoil surface. In section 3.3 discussions are made concerning the noise generation mechanisms by considering the frequency filtered fluctuating pressure field. Possible explanations are provided for the trends identified on the wall in the previous subsection. Finally a summary of the findings is provided in section 4 .

\section{Computational methodology}

The geometry considered in this paper is a NACA0012 aerofoil with a sharp trailing edge (TE) at free-stream Reynolds number $R e_{\infty}=50,000$ and Mach number $M_{\infty}=0.4$. We consider three different angles of attack $\alpha=5^{\circ}$ (laminar separation bubble), $\alpha=10^{\circ}$ (near-stall) and $\alpha=15^{\circ}$ (full-stall). Direct Numerical Simulations (DNS) are carried out in order to obtain the near-field hydrodynamic data, whereas

70 a Ffowcs-Williams and Hawkings solver based on surface integration is used to approximate the far-field noise.

\subsection{Governing equations and numerical methods}

The three-dimensional compressible Navier-Stokes equations with a source term, written in a conservative form in a generalized coordinate system and non-dimensionalised are:

$$
\frac{\partial}{\partial t}\left(\frac{\boldsymbol{Q}}{J}\right)+\frac{\partial}{\partial \xi_{i}}\left(\frac{\boldsymbol{E}_{j}-R e_{\infty}^{-1} M_{\infty} \boldsymbol{F}_{j}}{J} \frac{\partial \xi_{i}}{\partial x_{j}}\right)=-\frac{a_{\infty}}{L_{c}} \frac{\boldsymbol{S}}{J},
$$

where the indices $i=1,2,3$ and $j=1,2,3$ denote the three dimensions and $a_{\infty}$ is the ambient speed of sound. The vectors of the conservative variables, inviscid and viscous fluxes are given by

$$
\left.\begin{array}{c}
\boldsymbol{Q}=\left[\rho, \rho u, \rho v, \rho w, \rho e_{\mathrm{t}}\right]^{T}, \\
\boldsymbol{E}_{j}=\left[\rho u_{j},\left(\rho u u_{j}+\delta_{1 j} p\right),\left(\rho v u_{j}+\delta_{2 j} p\right),\left(\rho w u_{j}+\delta_{3 j} p\right),\left(\rho e_{\mathrm{t}}+p\right) u_{j}\right]^{T}, \\
\boldsymbol{F}_{j}=\left[0, \tau_{1 j}, \tau_{2 j}, \tau_{3 j}, u_{i} \tau_{j i}+q_{j}\right]^{T},
\end{array}\right\}
$$

with the stress tensor and heat flux vector written as

$$
\tau_{i j}=\mu\left(\frac{\partial u_{i}}{\partial x_{j}}+\frac{\partial u_{j}}{\partial x_{i}}-\frac{2}{3} \delta_{i j} \frac{\partial u_{i}}{\partial x_{i}}\right), \quad q_{j}=\frac{\mu}{(\gamma-1) \operatorname{Pr}} \frac{\partial T}{\partial x_{j}},
$$

where $x_{j}=\{x, y, z\}$ are the Cartesian coordinates, $\xi_{i}=\{\xi, \eta, \zeta\}$ are the generalized body fitted coordinates, $\delta_{i j}$ is the Kronecker delta, and the Jacobian determinant of the coordinate transformation is given by $J^{-1}=|\partial(x, y, z) / \partial(\xi, \eta, \zeta)|[10]$. Additionally, the total energy $e_{\mathrm{t}}=p /[(\gamma-1) \rho]+u_{j} u_{j} / 2$, where $\gamma=1.4$ for air and $u_{j}=\{u, v, w\}$. The local dynamic viscosity $\mu$ in (3) is calculated via Sutherland's law [11. In the above equations length scales are normalised by the aerofoil chord length $L_{c}$, velocities by the ambient speed of sound $a_{\infty}$, times scales by $L_{c} / a_{\infty}$ and pressure by $\rho_{\infty} a_{\infty}^{2}$. Temperature, density and dynamic viscosity are normalised by their respective ambient values: $T_{\infty}, \rho_{\infty}$ and $\mu_{\infty}$.

Characteristic-based non-reflecting boundary conditions (GCBCs) 12 are used at longitudinal and vertical far-field boundaries in order to prevent any reflections from outgoing waves. Furthermore, disturbances near the boundaries are reduced towards the required mean flow condition by implementation of a sponge zone included in the source term $S$ in (1) [13, 14. This term is non-zero within the sponge layer only, which is a perimeter two chord lengths thick around the longitudinal and vertical domain boundaries. On the aerofoil surface a characteristic based no-slip wall boundary conditions is used [15], while periodic boundary conditions are implemented in the spanwise direction. A moving frame technique is used to initialise the simulations which gradually ramps the velocity from zero to the free-stream value $\left(u_{\infty}, v_{\infty}, w_{\infty}\right)=(|\boldsymbol{u}| \cos (\alpha),|\boldsymbol{u}| \sin (\alpha), 0)$ over a period of $\Delta t^{*}=\Delta t a_{\infty} / L_{c}=5$ non-dimensional time units.

The governing equations are discretised using high-order accurate numerical methods specifically developed for aeroacoustic simulations on structured grids. The spatial flux derivatives are calculated with pentadiagonal compact finite difference schemes based on a seven-point stencil which maintain fourth-order 
accuracy at interior regions and at the boundaries [16. Numerical stability is maintained by implementing sixth-order pentadiagonal compact filters with a variable cut-off wavenumber. This is set to $\pi$ at the boundaries and $0.85 \pi$ in the interior regions [17. Time integration is carried out by using the classical fourth-order

${ }_{95}$ Runge-Kutta scheme with a CFL number of 0.95. Since the compact finite difference schemes and filters are implicit in space they require an inversion of a set of pentadiagonal coefficient matrices. This procedure requires a precise and efficient technique for parallel simulations in order to avoid numerical artefacts that may appear at the subdomain (processor core) boundaries. In the current paper a quasi-disjoint matrix systems [18] approach is selected offering super-linear scalability. The implementation is based on domain

100 decomposition and message passing interface (MPI) routines. Calculations are carried out on the IRIDIS5 cluster at the University of Southampton as well as the UK national supercomputer ARCHER. The grid is distributed onto 1200 processor cores for the prior case, and 6720 for the latter.

\subsection{Domain, geometry and meshing}

The computational domain is a rectangular cuboid with the aerofoil at the centre. As previously mentioned, the longitudinal and vertical boundaries of the domain are surrounded by the sponge layer through which any acoustic waves are attenuated and absorbed to prevent numerical reflections. The two regions of the domain; the inner region (physical domain) where meaningful simulation data are obtained; and, the sponge layer zone are defined as

$$
\left.\begin{array}{c}
\mathcal{D}_{\infty}=\left\{\boldsymbol{x} \mid x / L_{c} \in[-9,9], y / L_{c} \in[-9,9], z \in\left[-L_{z} / 2, L_{z} / 2\right]\right\}, \\
\mathcal{D}_{\text {phys }}=\left\{\boldsymbol{x} \mid x / L_{c} \in[-7,7], y / L_{c} \in[-7,7], z \in\left[-L_{z} / 2, L_{z} / 2\right]\right\}, \\
\mathcal{D}
\end{array}\right\}
$$

where $L_{c}$ denotes the aerofoil chord length, and $L_{z}$ is the spanwise domain length. The origin of the domain is at the centre, such that the aerofoil leading and trailing edges are located at $x / L_{c}=-1 / 2$ and $1 / 2$ respectively. The spanwise domain size is set to $L_{z}=0.2 L_{c}$ for the lowest angle of attack $\alpha=5^{\circ}$. At higher angles, particularly in the stall regime, a larger span is required in order to fully capture the extent of spanwise structures in the separated flow. In this paper a span length of $L_{z}=L_{c}$ is used for $\alpha=10^{\circ}$ and $15^{\circ}$, which has been verified to obtain converged results for far-field noise predictions in stall [19.

A H-topology grid system is used to decompose the domain into six blocks. The grid is stretched in the longitudinal and vertical directions, and is uniform in the spanwise direction. Extra refinement is included for the first two chord lengths above the aerofoil, which roughly corresponds to the wake height near the outflow boundary for the full-stall case. Similarly, a large proportion of the points in the streamwise direction are maintained in the first two chord lengths downstream of the TE in order to resolve the wake near the aerofoil. The smallest cells are clustered at the aerofoil leading and trailing edges. The number of grid cells used in each direction is $\left(N_{x}, N_{y}, N_{z}\right)=(1200,1120,326)$ (or $N_{z}=66$ for $\left.\alpha=5^{\circ}\right)$.

The grid is shown in figure 1 from three perspectives: full domain and grid with one in five points shown in each direction $(a)$, close up near the aerofoil with one in four points shown $(b)$, and aerofoil surface mesh $(c)$. Figure 1 $(a)$ and $(b)$ show how the grid is stretched asymmetrically in the vertical direction so that there is additionally refinement on the aerofoil suction side where transition to turbulence and separation occur. On the lower side a laminar boundary layer is maintained at the current Reynolds number. Additionally, in figure 1 $(a)$ the grid lines are coloured by vertical velocity (for $\alpha=15^{\circ}$ ), demonstrating the refinement in the downstream wake region. The instantaneous surface mesh sizes in wall units are provided in figure $2(a)$ averaged in span for $\alpha=10^{\circ}$ and $15^{\circ}$. For both cases the surface mesh sizes are below the recommended requirement suggested by Georgiadis et al. [20] for DNS: $10 \leq \Delta x^{+} \leq 20, \Delta y^{+}<1,5 \leq \Delta z^{+} \leq 10$.

In addition to the wall mesh sizes, the grid size away from the wall is analysed by comparing to the local Kolmogorov micro-scale $\eta=\left(\nu^{3} / \epsilon\right)^{1 / 4}$. This is particularly important for high angles of attack where highly turbulent flow is observed away from the aerofoil surface. The time averaged turbulence dissipation rate is calculated as $\epsilon=2 \nu \overline{\mathcal{S}_{i j} \mathcal{S}_{i j}}$, where $\mathcal{S}_{i j}$ is the rate of strain tensor. An approximate target value is obtained from the guidelines suggested by Laizet et al. 21 for DNS simulations of free turbulence. It was shown that spacings $\approx 5 \eta$ were sufficient to obtain a good agreement with experimental data for first and second order moments. Figure $2(b)$ shows a contour plot of the ratio between cube root of cell volume $J^{1 / 3}$, and 
(a)

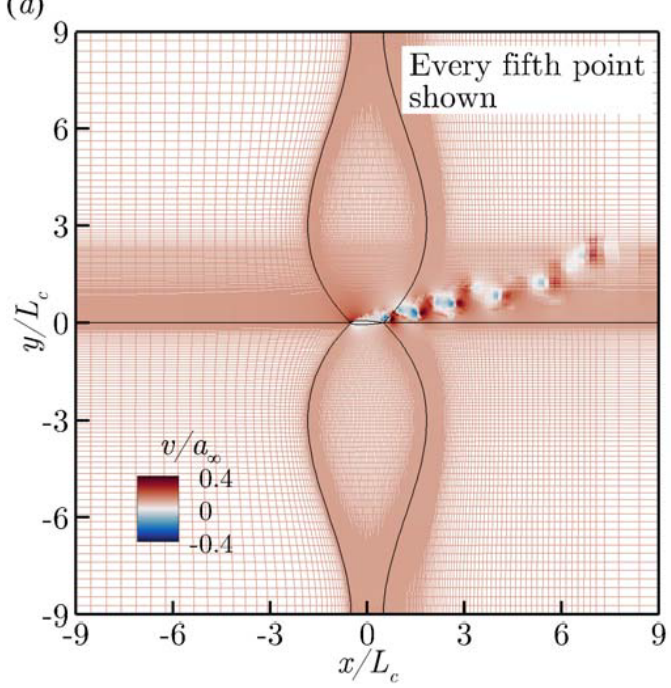

(b)

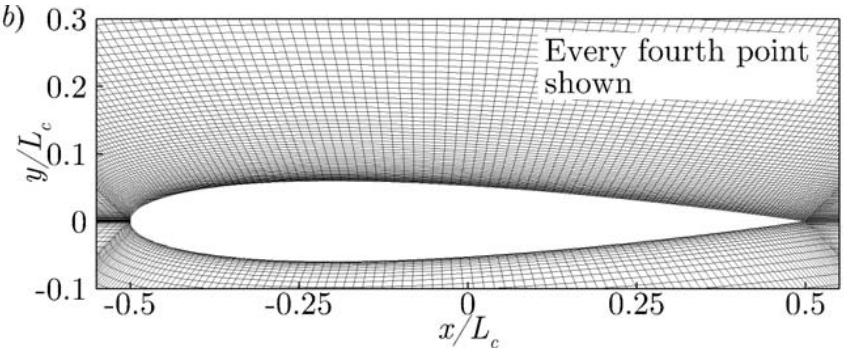

(c)

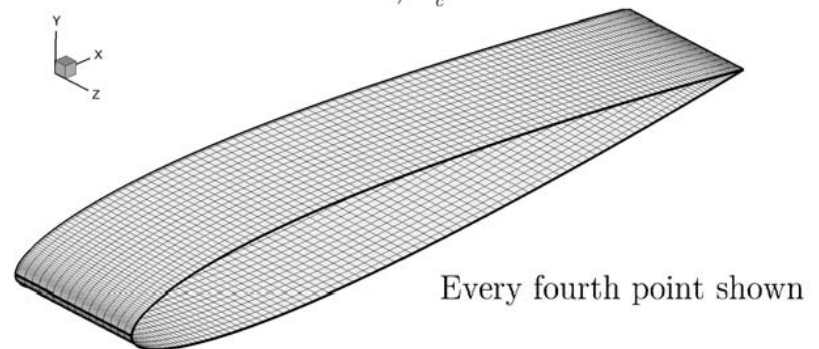

Figure 1: Computational mesh used in the numerical setup. (a) Side view of full domain with grid lines coloured by vertical velocity $v / a_{\infty}$ (every fifth point shown in each direction). (b) Close up of NACA0012 profile (every fourth point shown in each direction). (c) Aerofoil surface mesh (every fourth point shown in each direction).

the Kolmogorov microscale on the aerofoil suction side for $\alpha=10^{\circ}$. The majority of the turbulent region satisfies $J^{1 / 3}<4 \eta$, with a maximum value around $5.5 \eta$.

\subsection{Far-field extrapolation approach}

A time domain solution to the Ffowcs-Williams and Hawkings equation [22] based on the Farassat 1A formulation [23, simplified for the current problem, is implemented in order to calculate the acoustic field.

$$
4 \pi p_{a}(\boldsymbol{x}, t)=\int\left[\frac{i}{a_{\infty} r\left(1-M_{r}\right)^{2}}+\frac{l-p M_{i} n_{i}}{r^{2}\left(1-M_{r}\right)^{2}}+\frac{\left(M_{r}-M^{2}\right) l}{r^{2}\left(1-M_{r}\right)^{3}}+\frac{\rho_{\infty} a_{\infty} u_{i} n_{i}\left(M_{r}-M^{2}\right)}{r^{2}\left(1-M_{r}\right)^{3}}\right]_{r e t} \mathrm{~d} S
$$

where $l=p \cos (\Phi)$, subscript $r e t$ indicates variables are analysed at the retarded time $\tau=t-r / a_{\infty}$, dotted variables indicate the time derivative, and $r$ is the effective acoustic distance calculated from the Garrick triangle 24 extended for two-dimensional mean flow velocity:

$$
r=\frac{M_{x} d_{x}+M_{y} d_{y}+\sqrt{\left(M_{x} d_{x}+M_{y} d_{y}\right)^{2}+\left(1-M_{x}^{2}-M_{y}^{2}\right)\left[d_{x}^{2}+d_{y}^{2}+d_{z}^{2}\right]}}{1-M_{x}^{2}-M_{y}^{2}},
$$

where $\left(M_{x}, M_{y}\right)=\left(M_{\infty} \cos (\alpha), M_{\infty} \sin (\alpha)\right)$ and $\left(d_{x}, d_{y}, d_{z}\right)=\left(x_{o}-x_{s}, y_{o}-y_{s}, z_{o}-z_{s}\right)$, with subscript $o$ and $s$ representing observer and source respectively. Additionally, $M_{r}=\boldsymbol{M} \cdot \hat{\boldsymbol{r}}$, where $\boldsymbol{r}$ is the effective radiation vector, and ${ }^{\wedge}$ represents a unit length. The local radiation angle $\Phi$, is determined by $\Phi=$ $\operatorname{acos}(\boldsymbol{n} \cdot \hat{\boldsymbol{r}})$, where $\boldsymbol{n}$ is the surface normal. The focus of the current paper is the aerofoil dipole sound. The above integration is therefore performed on the aerofoil surface. This neglects direct quadrupole noise, although some signature of quadrupole sound scattered by the wall may be present. The impenetrable surface integration greatly reduces the cost of the calculation, requiring only the pressure as an input variable.

The spanwise periodic boundary condition implemented in the simulations is also applied in the far-field noise calculation. In order to achieve this the surface integral is performed iteratively on domains shifted $n L_{z}$ either side of the aerofoil. This process is repeated until a converged solution is obtained, which occurs due to $r$ increasing and $\Phi \rightarrow \pi / 2$ as the $z$ coordinate is increased. For the current setup a span of 20 chord lengths was found to be sufficient for converged far-field spectra results. Validation of the current FW-H implementation is included in [25]. 


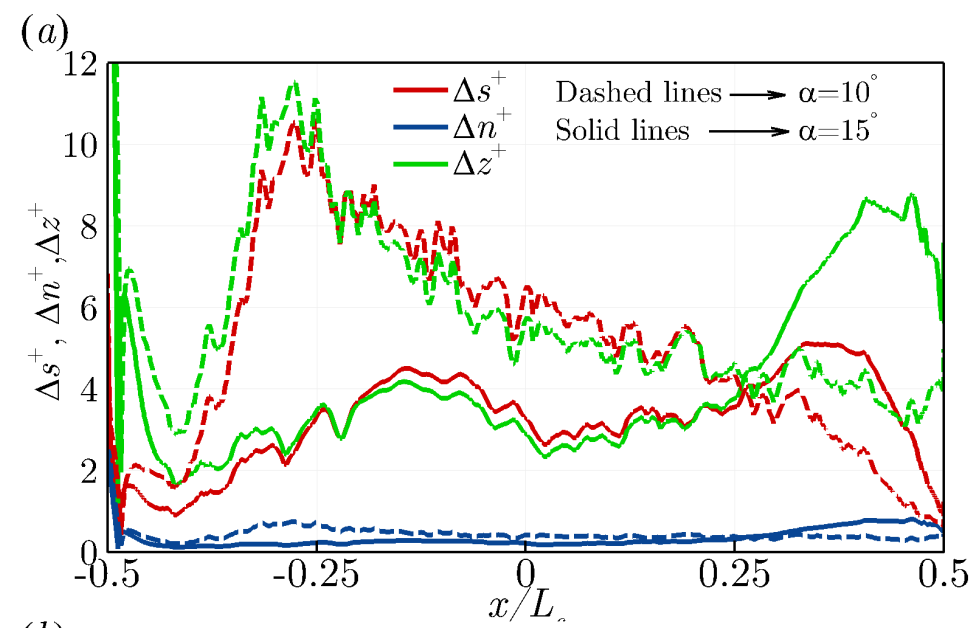

(b)

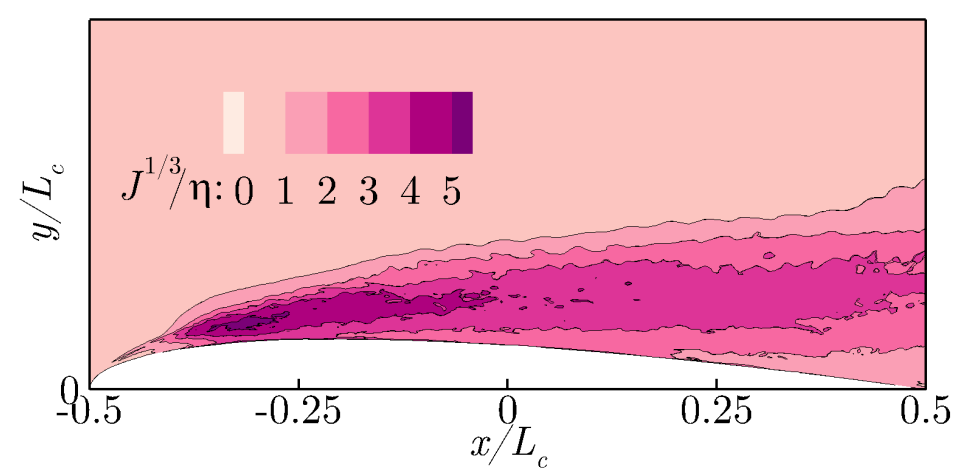

Figure 2: (a) Instantaneous grid sizes in wall units over the aerofoil surface averaged in span at $t a_{\infty} / L_{c}=200 . \Delta s$ and $\Delta n$ represent the body fitted and wall normal spacings respectively. (b) Cube root of cell volume compared to the Kolmogorov microscale in the $x y$-plane (averaged in span) for $\alpha=10^{\circ}$.

\subsection{Definition of variables for statistical analysis}

Data processing and analysis are carried out upon the completion of each simulation. The far-field (acoustic) pressure is defined as:

$$
p_{a}(\boldsymbol{x}, t)=p(\boldsymbol{x}, t)-\bar{p}(\boldsymbol{x}),
$$

where $\bar{p}(\boldsymbol{x})$ is the time averaged pressure. Following the definitions used in [26], the Fourier transform of $p_{a}$ can then be calculated as

$$
P_{a}(\boldsymbol{x}, f, T)=\int_{-T}^{T} p_{a}(\boldsymbol{x}, t) e^{2 \pi i f t} \mathrm{~d} t
$$

and the one-sided frequency based PSD function as:

$$
S_{p p a}(\boldsymbol{x}, f)=\lim _{T \rightarrow \infty} \frac{P_{a}(\boldsymbol{x}, f, T) P_{a}^{*}(\boldsymbol{x}, f, T)}{T},
$$

where ' $*$ ' denotes a complex conjugate. The same definitions can also be used to calculate the spectra of other variables. The sound power spectra is also calculated by integrating the fluctuating pressure PSD between two observer angles $\theta_{1}$ and $\theta_{2}$ :

$$
W_{p p a}\left(\theta_{1}, \theta_{2}, f\right)=\frac{L R}{\rho_{\infty} a_{\infty}} \int_{\theta_{1}}^{\theta_{2}} S_{p p a}(\theta, f) d \theta
$$


where $R$ is the distance to the observer from the aerofoil mid-chord, and $L$ is the span of the integration surface, taken as $20 L_{c}$ for consistency with the FW-H calculations.

The acoustic sources are investigated by considering the surface distribution of the leading term in the FW-H equation (5). By considering contributions from both sides of the aerofoil, and using far-field observer assumptions $\left(r \gg L_{c}\right)$, the Fourier transform of this term can be simplified to $-i \omega L_{w} /\left(a_{\infty} r\left(1-M_{r}\right)^{2}\right)$, where

$$
L_{w}\left(x, z, \boldsymbol{x}_{o}, f, T\right)=\int_{-T}^{T} l_{w}\left(x, z, \boldsymbol{x}_{o}, t\right) e^{2 \pi i f t} \mathrm{~d} t,
$$

and

$$
l_{w}\left(x, z, \boldsymbol{x}_{o}, t\right)=l\left(x, y_{u}, z, \boldsymbol{x}_{o}, t\right)+l\left(x, y_{l}, z, \boldsymbol{x}_{o}, t\right),
$$

with subscripts $u$ and $l$ indicating the upper and lower sides of the aerofoil respectively. For an observer in the far-field the terms $\omega, r$, and $M_{r}$ may be considered constant over the aerofoil surface. We therefore consider the term $L_{w}$ in order to analyse the distribution of sources over the wall at a given frequency.

\section{DNS results}

The numerical simulations are run with an adaptive time step of approximately $\Delta t^{*}=1 \times 10^{-4}$ for 60 (for $\alpha=15^{\circ}$ ) and 200 (for $\alpha=10^{\circ}$ and $15^{\circ}$ ) non-dimensional time units respectively. Data acquisition is conducted with a sampling rate of $\Delta t^{*}=0.02$ providing 1000 flow snapshots over the final 20 time units of each simulation. Processed frequency domain data is produced with a tapered cosine (Tukey) window utilising Welch's averaging technique with three equal sized $50 \%$ overlapping windows.

Instantaneous snapshots of the flow and the acoustic near-field are shown in figure $3(a)-(c)$ for the three cases. The flow field is shown by Q-criterion iso-surfaces for $Q=20$ coloured by velocity magnitude, while the sound is visualised by divergence of velocity $\nabla \cdot \boldsymbol{u}$ contours taken in the $z=0$ plane. For the current condition the aerofoil undergoes a leading edge (LE) separation process as the angle of attack is increased. Firstly a laminar separation bubble is formed at low angle of attack, which is shifted upstream for higher angles until it eventually bursts resulting in a fully stalled flow. The time and spanwise averaged pressure $\left(C_{p}\right)$ and skin friction $\left(C_{f}\right)$ coefficients are shown in figure $4(a)$ and $(b)$ respectively. At $\alpha=5^{\circ}$ laminar separation occurs at around $10 \%$ of the chord, and reattaches as a turbulent boundary layer at around $60 \%$ of the chord. Comparison is made with the DNS data obtained by Jones et al. 27. Overall there is a good match for both quantities on both suction and pressure sides. At $\alpha=10^{\circ}$ the boundary layer separates at the aerofoil LE. Transition of the separated shear layer occurs through the Kelvin-Helmholtz instability process and reattaches at around $x / L_{c}=-0.225$, resulting in a significantly shorter bubble than the lower angle of attack. Downstream of this location a turbulent boundary layer remains attached over the majority of the surface, although some trailing edge separation can be observed at certain instances of time. At (150 aerofoil is fully stalled, which is apparent from the collapse of the suction peak in figure $4(a)$. The main characteristic of the flow is large scale shedding in the wake similar to that encountered by a bluff body. This occurs when vortices shed from the separated shear layer on the aerofoil suction side pair with vortices shed from the TE, resulting in a von Kármán vortex street.

Regarding the sound field, figure 3 highlights significant differences in the acoustic near-field for low and

high angle of attack cases. At $\alpha=5^{\circ}$ a mainly dipolar sound wave pattern is shown, emanated primarily from the TE. Some interference patterns are also observed on the aerofoil suction side which are caused by quadrupole sources in the turbulent boundary layer occurring at moderate Mach numbers. In comparison for $\alpha=10^{\circ}$ and $15^{\circ}$ waves clearly emanate from multiple sources in the shear layer and the wake as well as the aerofoil TE. This indicates that the noise generation at high angle of attack occurs through different physical mechanisms to low angles which are thoroughly documented. At $\alpha=10^{\circ}$ the majority of the sound appears to radiate from the transition region near the LE, and contains noticeably higher frequency components (especially compared to $\alpha=5^{\circ}$ ). At $\alpha=15^{\circ}$ noise radiated directly by the wake seems to have a similar prominence to sound waves generated nearer to the aerofoil surface. This is most clear downstream on the aerofoil pressure side where waves can be observed convecting upstream. It is worth noting that in both high 


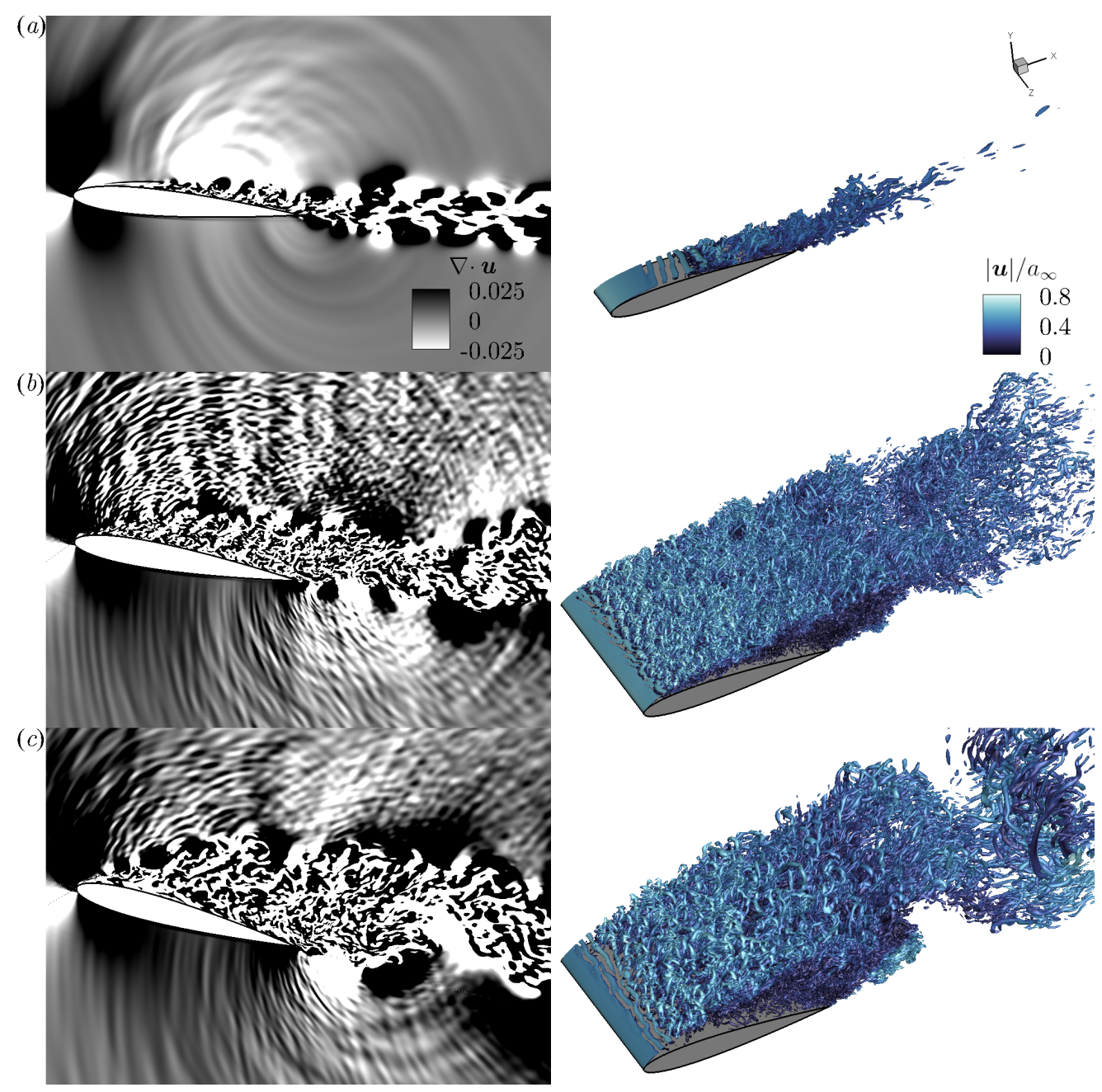

Figure 3: (a) Divergence of velocity $\nabla \cdot \boldsymbol{u}$ contours shown in the $x y$-plane for the three angles of attack. (b) Iso-surfaces of $\mathrm{Q}$-criterion $(\mathrm{Q}=20)$ coloured by velocity magnitude $|\boldsymbol{u}| / a_{\infty}$. Snapshots are taken the end of each simulation.

(a)

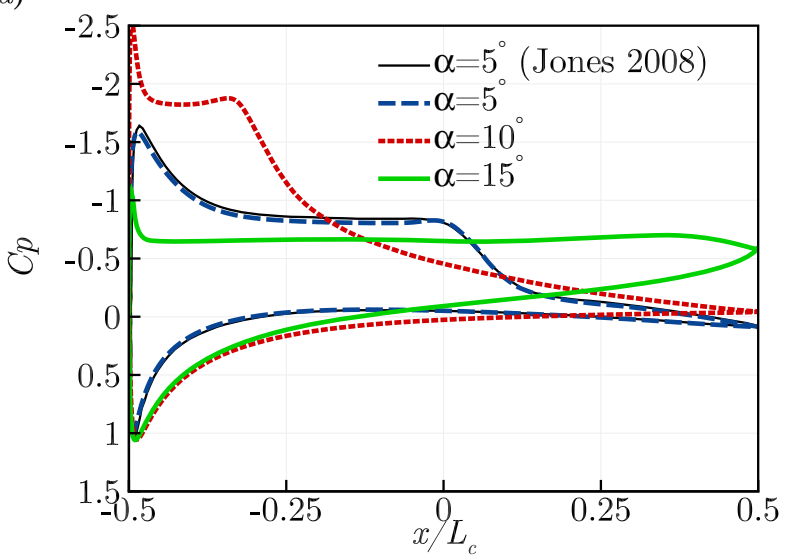

(b)

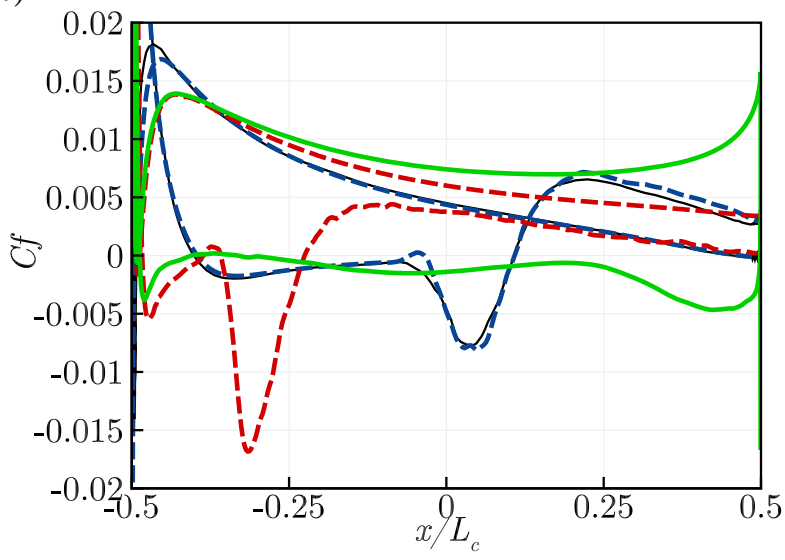

Figure 4: Time averaged pressure $C_{p}(a)$ and skin friction $C_{f}(b)$ coefficients compared for the three angles of attack. 
and therefore strong compressibility effects are anticipated. The current results indicate that both dipole and quadrupole sources may play a role under stall conditions. Despite this, previous experimental studies (5, 7]) have suggested a predominantly dipolar radiation. It has been shown previously that at moderate Mach numbers direct quadrupole noise may also contribute to high frequency noise [28], it is likely this is not observed in the previous experimental work due to the limited speed achievable in open jet wind tunnel facilities. In the current study we focus on analysis of the dipole noise sources, which are expected to dominate at low frequencies where stall noise has been seen to be most significant, and is more relevant to the understanding of previous experimental works. A follow on study by the authors will investigate the effect of high frequency quadrupole sources in detail.

\subsection{Radiated spectra and directivity trends}

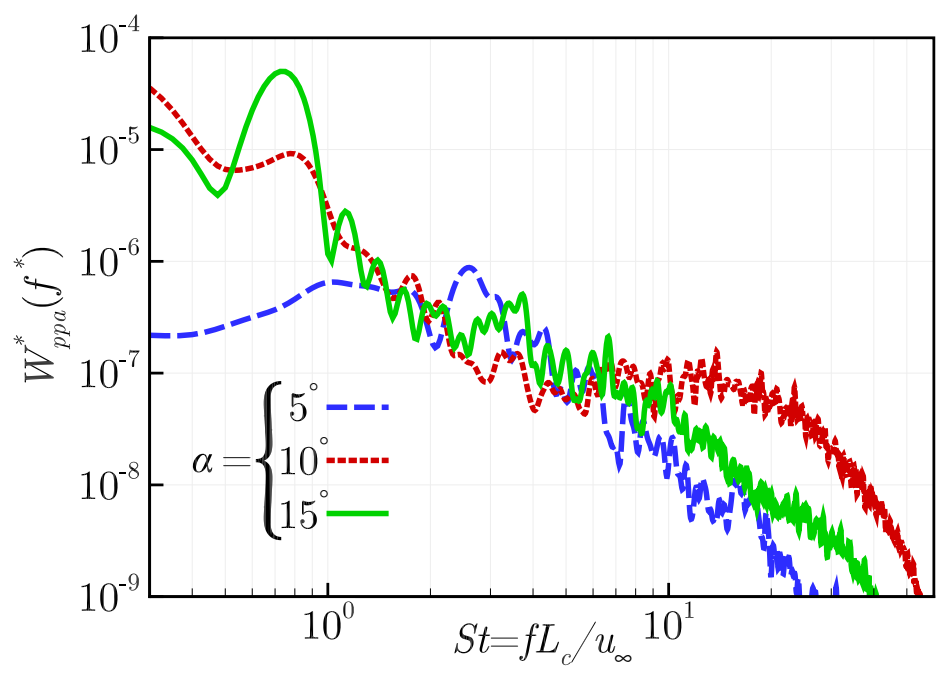

Figure 5: Sound power (normalised) (see 10 ) obtained ten chords from the aerofoil mid-chord calculated for $0 \leq \theta<360^{\circ}$.

The sound power spectra obtained ten chord lengths from the aerofoil mid-chord over $0 \leq \theta<360^{\circ}$ is provided in figure 5 for the three angles of attack. Three distinct frequency bands are observable. Firstly, low frequency $S t \leq 1.5$ where the high angle cases dominate. Both $\alpha=10^{\circ}$ and $15^{\circ}$ attain a broadband increase of up to $20 \mathrm{~dB}$ relative to the $\alpha=5^{\circ}$. Additionally, as suggested by Moreau et al. [5] narrow band peaks are also observed for the full-stall case. The dominant peak occurring at $S t=0.75$ corresponds to the von Kármán shedding in the wake. The second frequency band, medium frequencies, occurs from $1.5<S t<10$. In this range the radiated sound power is comparable for all three cases, except within a narrow band where a peak occurs for the $\alpha=5^{\circ}$ case centred at $S t=2.60$. Finally, for high frequencies $S t \geq 10$, an increase in sound power of up to $15 \mathrm{~dB}$ is shown for $\alpha=10^{\circ}$ relative to the $\alpha=5^{\circ}$ case, and $\mathrm{dB}$ relative to the $\alpha=15^{\circ}$ case. This observation has not been made previously, and may be linked to the specific flow conditions. For example scattering of wake quadrupole noise at the TE, or scattering of noise from the separated shear layer at the LE, may play an important role to the high frequency dipole prediction when the Mach number is high enough.

The effect of observer angle on the radiated noise is shown by the sound directivity plots in figure 6 . The data is based on Fourier transform magnitude of the acoustic pressure $\left|P_{a}\right|$, given in third octave bands. The three angles of attack are contrasted for six Strouhal numbers: $(a) S t=0.75,(b) S t=1.13,(c) S t=2.0$, (d) $S t=4.0,(e) S t=8.0$, and $(f) S t=16.0$. In the current plot the observer angle $\theta$ is defined relative to the aerofoil chord line. This provides the fairest comparison between the different cases since increasing the angle of attack tends to rotate the dipole pattern clockwise when observer angles are considered relative to the mean flow direction. The first two Strouhal numbers correspond to the first a second peaks occurring 
(a) $S t=0.75$

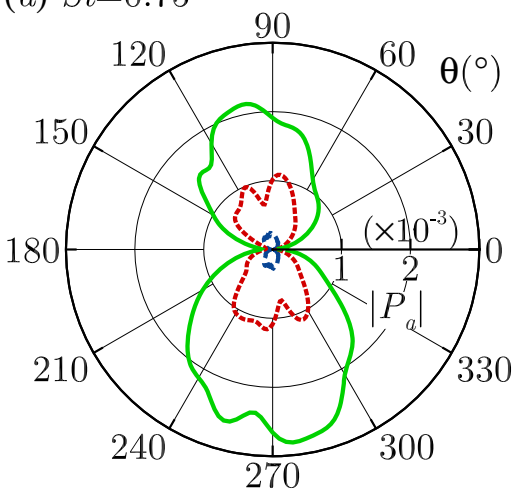

(d) 4.00

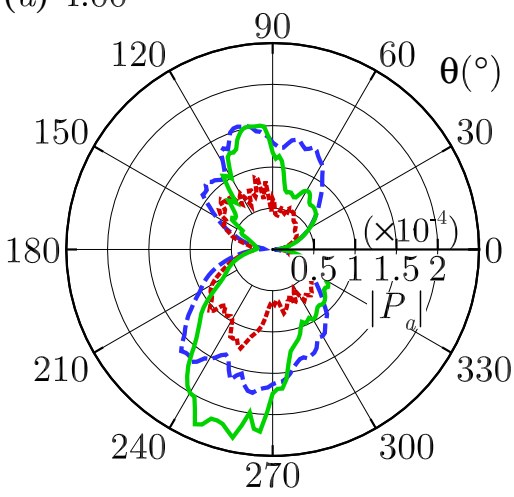

(b) 1.13

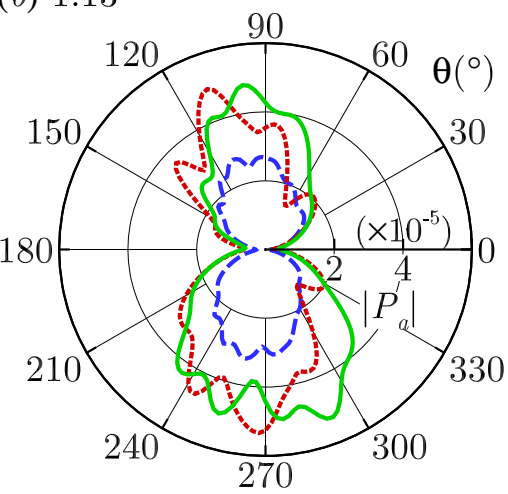

(e) 8.00

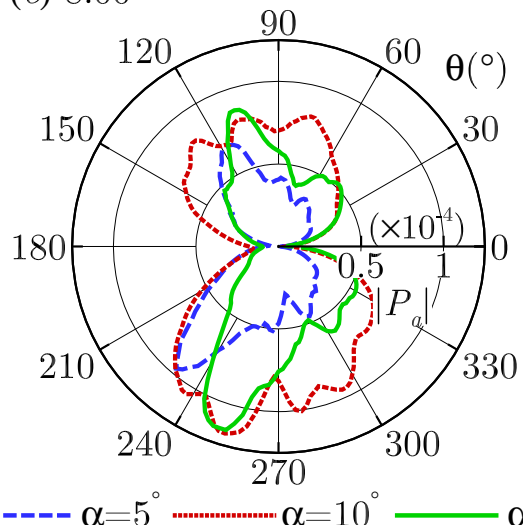

(c) 2.00

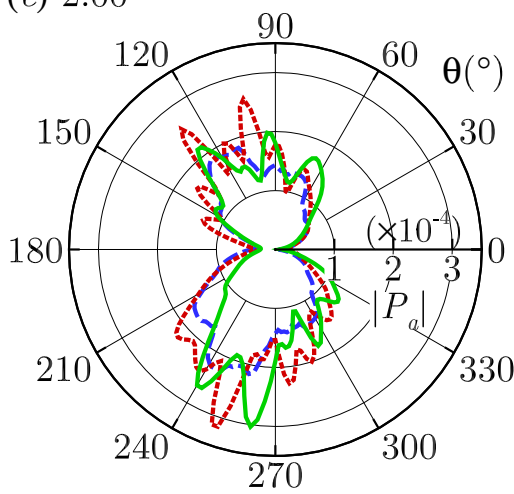

(f) 16.00

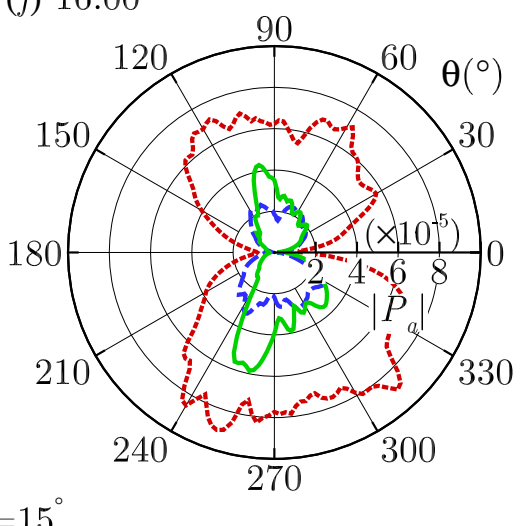

Figure 6: 1/3 octave band sound directivity based on magnitude of acoustic pressure Fourier transform $\left(\left|P_{a}\right|\right)$ at observers $\left(x_{o}, y_{o}, z_{o}\right)=\left(10 L_{c} \cos (\theta), 10 L_{c} \sin (\theta), 0\right)$. Results are shown for six Strouhal numbers $\left(S t=f L_{c} / u_{\infty}\right)$ and the three angles of attack.

for the $\alpha=15^{\circ}$ case in figure 5. At $S t=0.75$ each angle of attack produces the expected dipolar radiation pattern, although there is increased asymmetry for the $\alpha=15^{\circ}$ case which produces a higher noise level in the lower half-plane. The increased noise level for $\alpha=15^{\circ}$ is observed in all observer directions suggesting the tone shown previously is not dependent on observer angle. As the frequency is increased up until $S t=2.0$ the directivity patterns become more comparable. In the medium frequency band $(S t=2.0$, 4.0, and 8.0) a more cardioid pattern is observed for each angle of attack, suggesting radiation of compact sources predominately near the TE. At $S t=4.0$ the least noise is produced by the $\alpha=10^{\circ}$ case. $\alpha=5^{\circ}$ and $\alpha=15^{\circ}$ obtain a similar maximum amplitude in the upper half-plane, while in the lower half-plane the $\alpha=15^{\circ}$ case dominates. The $\alpha=15^{\circ}$ case is also more directional than $\alpha=5^{\circ}$ with main lobes at $\theta=100^{\circ}$ and $255^{\circ}$. At $S t=8.0$ the largest noise signatures are recorded for $\alpha=10^{\circ}$ and $\alpha=15^{\circ}$, although the $\alpha=10^{\circ}$ case shows less directional bias, with increased levels in the upstream and downstream directions. The lack of cardioid pattern for $\alpha=10^{\circ}$ may suggest significant radiation occurring from both near the LE and the TE. The directivity pattern for $\alpha=15^{\circ}$ is very similar to what is observed for the lower frequency of $S t=4.0$. A similar pattern is also observed for $\alpha=5^{\circ}$, although the noise generated is less and the main lobes are pointed further upstream. The directivity at $S t=16.0$ in figure $5(f)$ shows that the increase in high frequency noise for $\alpha=10^{\circ}$ is observed in all observer directions. The largest increases relative to the full-stall case are attained in the upstream half-plane at approximately $\theta=120^{\circ}$ and $230^{\circ}$. It is also interesting to note that the full-stall case maintains a strong directional bias, although the other two cases radiate more uniformly.

The sound power spectra is also shown in figure 7 calculated over a narrower $12^{\circ}$ observer angle range 


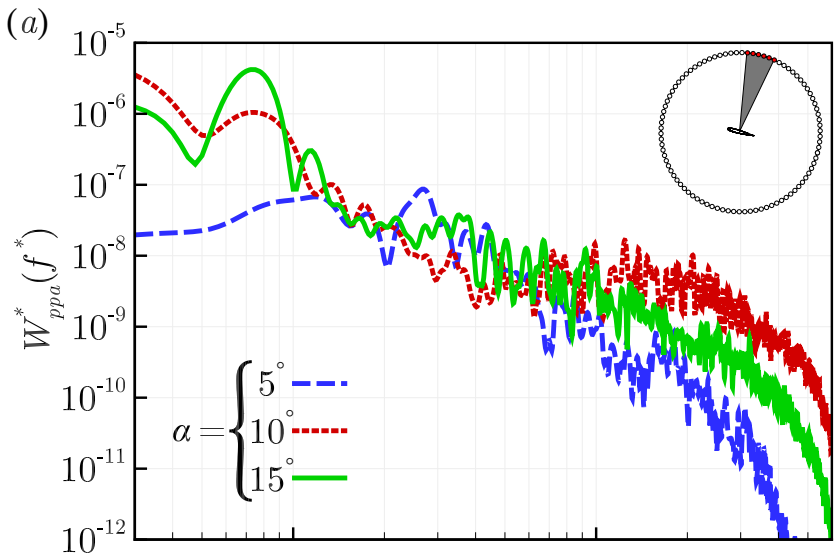

(b)
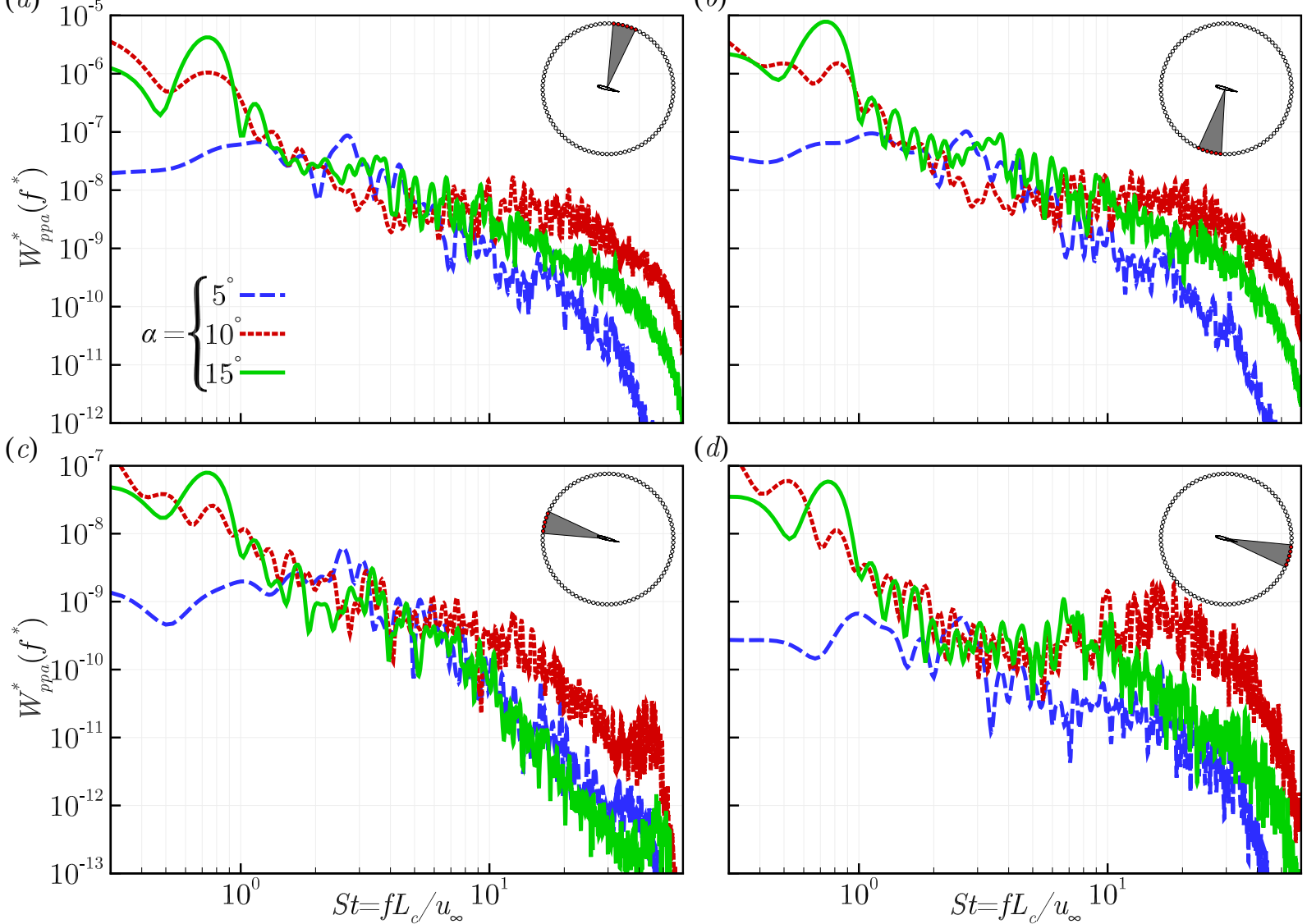

(d)

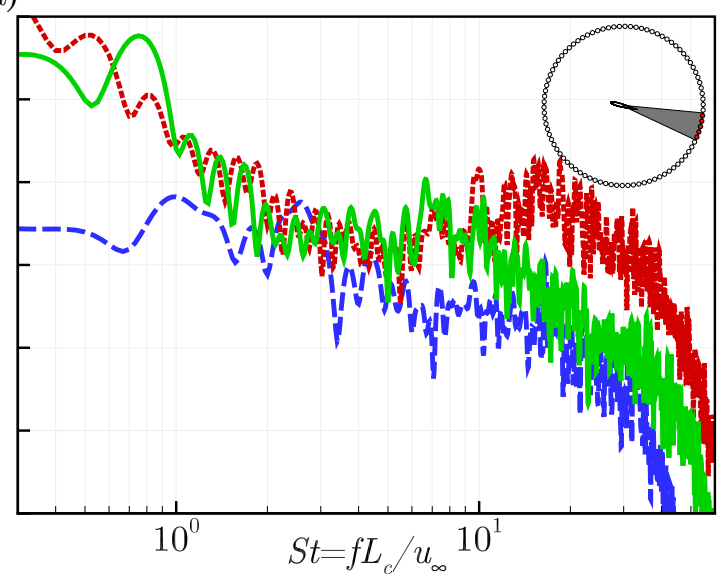

Figure 7: Sound power (normalised) calculated over a $12^{\circ}$ observer band $\left(\theta-6^{\circ} \leq \theta \leq \theta+6^{\circ}\right)$ at four central observer locations. (a) Directly overhead of the aerofoil $\theta=90^{\circ}$. (b) Directly below $\theta=270^{\circ}$. (c) Upstream $\theta=180^{\circ}$. ( $d$ ) Downstream $\theta=0^{\circ}$.

centred at four locations: $(a)$ directly above the aerofoil $\theta=90^{\circ},(b)$ directly underneath $\theta=270^{\circ},(c)$ upstream $\theta=180^{\circ},(d)$ downstream $\theta=0^{\circ}$. A similar frequency distribution is observed both directly above and below the aerofoil, except for an increase in low frequency noise on the aerofoil underside, mentioned previously. The sound spectra differs significantly for observers directly upstream and downstream of the aerofoil, especially at medium-high frequencies. Compared to the vertical observer angles, there is a larger increase in high frequency noise attained for $\alpha=10^{\circ}$ relative to $\alpha=15^{\circ}$. By considering the FW-H equation (5) we can speculate that this difference must occur due to sound sources appearing in the vicinity of the LE. This is because the term $\cos (\Phi)$ is greatest at the LE for horizontal observer angles, and weakest at the LE for vertical observer angles. The remainder of this paper is aimed at identifying some of the underlying causes for the differences observed for radiated noise identified in this section. In particular the differing trends observed at low $(S t \leq 1.5)$, medium $(1.5<S t<10)$ and high $(S t \geq 10)$ frequency bands.

\subsection{Dipole source identification}

In this section the acoustic sources on the aerofoil surface are investigated in detail in order to provide explanations for the trends identified earlier. As mentioned previously, the source is visualised by considering the part of the leading term in the FW-H equation which varies over the surface (see section 2.4 for the full explanation). In figure 8, contours of $L_{w}$ (see (11) ) are plot over the aerofoil upper surface for an observer located at $\theta=90^{\circ}$ and $R=10 L_{c}$, corresponding to figure $7(a)$. The result is also relevant for $\theta=270^{\circ}$ by considering that $L_{w}(\theta) \approx-L_{w}\left(\theta+180^{\circ}\right)$. The figure shows Fourier transform magnitude $\left|L_{w}\right|$ in a $\log$ scale 
and the imaginary part of Fourier transform $\operatorname{Imag}\left(L_{w}\right)=\left|L_{w}\right| \sin (\phi)$, where $\phi$ is the phase angle. The prior is used to visualise the amplitude of fluctuations, while the latter also shows the phase variations over the surface. (The real part of Fourier transform could also be used to analyse the phase, but is not considered here for succinctness.) It is worth noting that high strength magnitude doesn't not guarantee noise radiates from a location, just that pressure fluctuations are large. This is because phase interferences may result in a negligible contribution in the FW-H integration. Nonetheless with both magnitude and phase information we can make inferences about the noise generation. Strouhal numbers of $S t=0.75$ and $S t=2.75$ are considered in figure $8(a)$ and $(b)$, respectively. At $S t=0.75$, the $\alpha=5^{\circ}$ and $10^{\circ}$ cases have a maximum source amplitude towards the end of the separation bubble where the separated shear layer transitions, just before boundary layer reattachment. Meanwhile, the $\alpha=15^{\circ}$ case exhibits high source strength over the majority of the aft portion of the aerofoil. This is consistent with the correlation data obtained by Paterson et al. 3], which indicated that an area upstream of the TE may act as a significant acoustic source under stall conditions. The high strength aft region is likely due to the pressure signature induced by shedding of large scale von Kármán vortices, and is also seen to some extent for the $\alpha=10^{\circ}$ case. Regarding the phase, the full-stall case shows an in-phase source relationship for almost the entire aerofoil surface. Comparatively, there is more variation for the pre-stall cases. For example, at $\alpha=10^{\circ}$ the separation bubble and TE regions are in-phase, however there is a moderate strength out-of-phase region appearing once the boundary layer reattaches. The far-field broadband increase observed for both high angle of attack cases at low frequency is likely due to a higher percentage of the surface exhibiting significant source strength. Meanwhile, the dominant tone occurring for $\alpha=15^{\circ}$ is caused by the consistently in-phase source area. At the frequency of $S t=2.75$ the $\alpha=5^{\circ}$ and $15^{\circ}$ cases show a similar radiated noise level which is slightly increased relative to $\alpha=10^{\circ}$ (see figure $7(a)$ and $(b)$ ). For the two pre-stall cases with separation bubbles, there is a high magnitude occurring at transition and downstream where there is an attached turbulent boundary layer. In the stall case the highest strength fluctuations are concentrated in two locations, near the TE, and a spanwise strip at around quarter chord. The upstream hotspot is likely caused by flow structures shed in the shear layer, while they are still close enough to the wall to induce significant pressure fluctuations. The Fourier transform magnitude indicates that the stall case should radiate less noise at this frequency, which appears to contradict the observations made in the far-field. However, this discrepancy can be rectified by also considering the phase distribution over the surface. The $\alpha=5^{\circ}$ and $10^{\circ}$ cases show a large amount of phase variation, whereas at $15^{\circ}$ most of the aft part of the aerofoil surface, and the high strength shear layer region remain in phase. This will result in more cancellations for the lower angle of attack cases when the data is integrated in the FW-H solver.

The same analysis is carried out for $S t=5.00$ and $S t=20.00$ in figure $9(a)$ and $(b)$. At $S t=5.00$ a similar source magnitude distribution is shown as at $S t=2.75$ for all three angle of attack cases. However, there are significant differences occurring when visualising the phase, which for each angle of attack varies more in a given area due to the smaller length scales associated with the higher frequency. Despite this, it is clear how relatively the phase changes more gradually for $\alpha=15^{\circ}$ compared to the other two angles of attack, indicating a more in-phase source relationship. This might explain how it is possible for the stall case to obtain a similar level of radiated noise despite lower maximum amplitude fluctuations on the wall. One possible explanation for reduced phase variation in stall could be a higher convection speed of the dominant flow structures if appear in the separated shear layer, compared to in an attached boundary layer for lower angles of attack.

A similar source distribution is shown again at a high frequency of $S t=20.00$ for $\alpha=5^{\circ}$ and $10^{\circ}$. The full-stall case $\left(\alpha=15^{\circ}\right)$ differs by no longer exhibiting a high strength region near the LE. At this frequency the highest sound levels are obtained for $\alpha=10^{\circ}$, which might be expected from the high magnitude observed over a large proportion of the surface. However, what is less clear is why the $\alpha=15^{\circ}$ case also obtains a high sound level, exceeding the $\alpha=5^{\circ}$ case, despite very low amplitude levels. As with lower frequency, it can be speculated that the more gradual variation of phase patterns for $\alpha=15^{\circ}$ may result in less destructive interference. However, this is less apparent from the qualitative contour plots as previously. We can verify the influence of the phase on the radiated noise by integrating $L_{w}$ over the aerofoil surface, then taking the magnitude squared in order to be dimensionally consistent with the far-field sound spectra. This procedure yields values of $\left|\int L_{w} d S\right|^{2}=3.8 \times 10^{-12}, 3.5 \times 10^{-10}$ and $1.5 \times 10^{-11}$ for $\alpha=5^{\circ}, 10^{\circ}$ and 
(a)
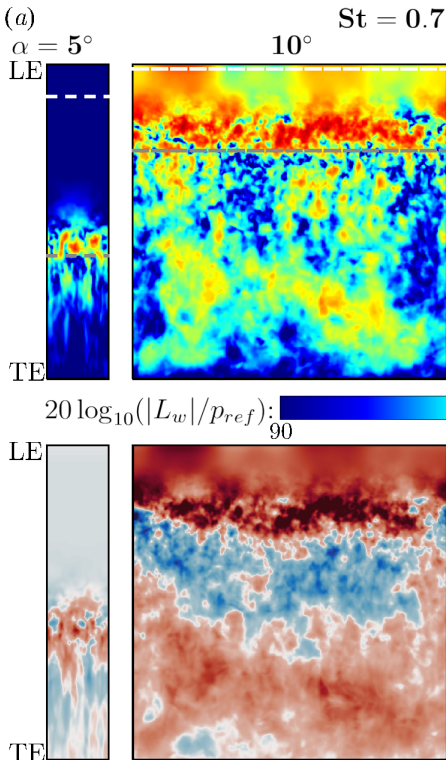

$\left|L_{w}\right| \sin (\phi) / p_{\infty}:-0.04$ $15^{\circ}$
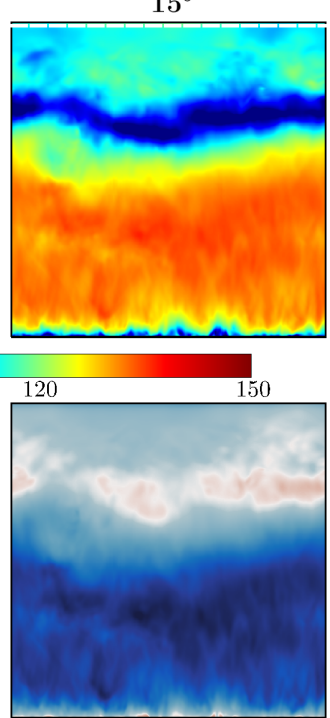

0.04 (b)
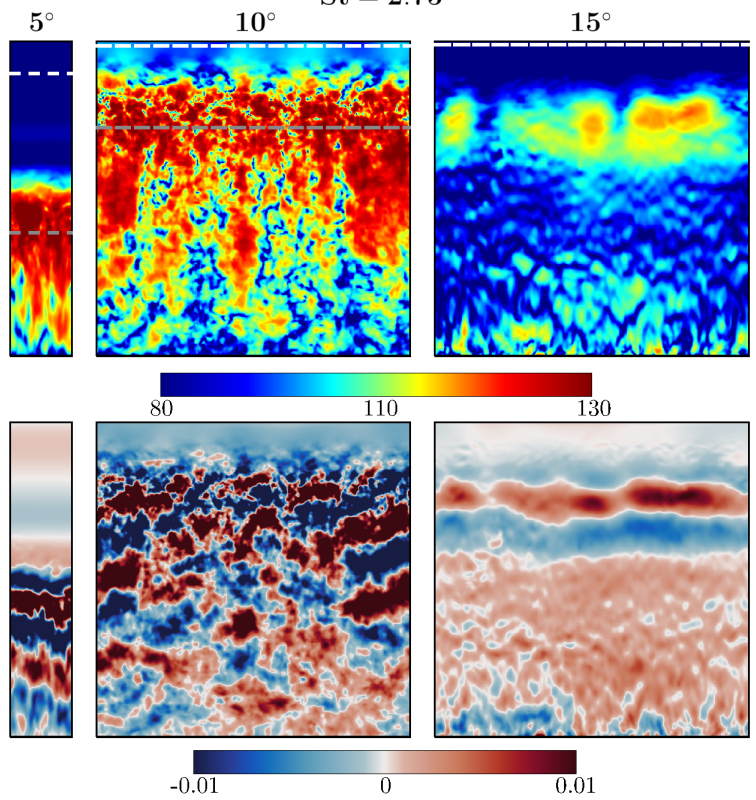

Figure 8: Fourier transform of $l_{w}$ (see 11) for the three angles of attack for an observer at $\left(x_{o}, y_{o}, z_{o}\right)=\left(0,10 L_{c}, 0\right)$. Two frequencies are shown: $S t=0.75(a), S t=2.75(b)$. The upper row shows Fourier transform magnitude (in logarithmic scale), while the lower row shows the imaginary part of Fourier transform. The reference pressure used is $p_{r e f}=10^{-10} p_{\infty}$. White and grey dashed lines represent the mean separation and reattachment points respectively.
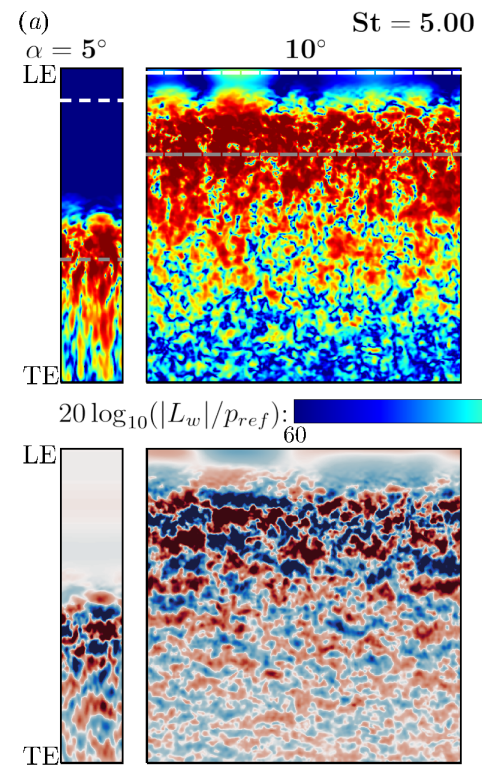

$\left|L_{w}\right| \sin (\phi) / p_{\infty}:-0.01$
$\mathrm{St}=5.00$
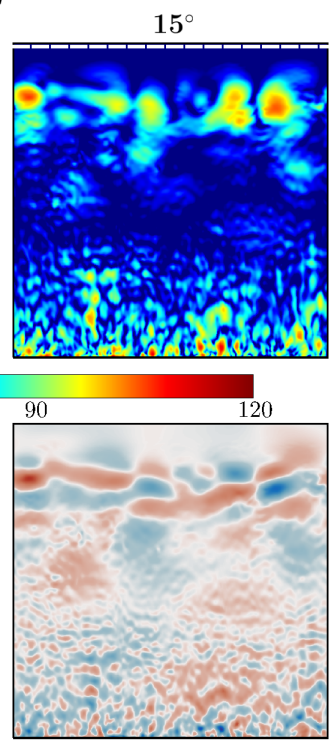

0 (b)
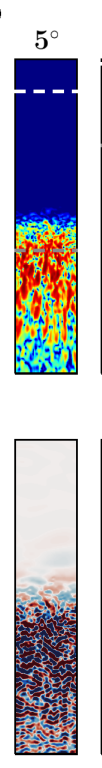
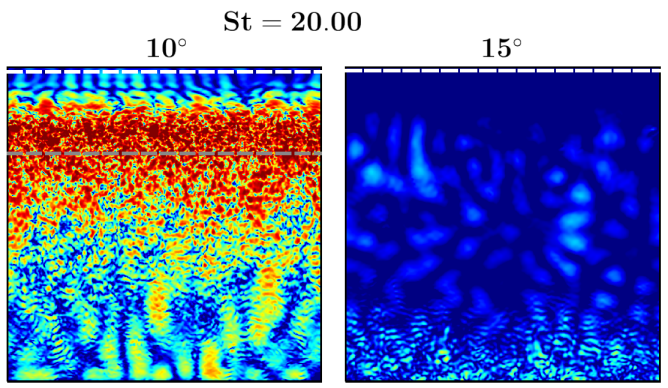

40

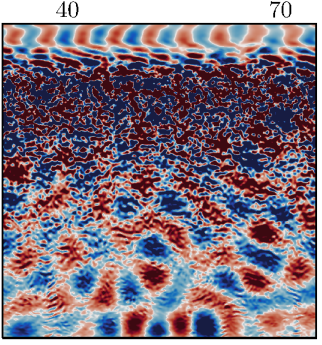

$-0.001$

\section{等}

0

Figure 9: Fourier transform of $l_{w}$ (see (11) for the three angles of attack for an observer at $\left(x_{o}, y_{o}, z_{o}\right)=\left(0,10 L_{c}, 0\right)$. Two frequencies are shown: $S t=5.00(a), S t=20.00(b)$. The upper row shows Fourier transform magnitude (in logarithmic scale), while the lower row shows the imaginary part of Fourier transform. The reference pressure used is $p_{r e f}=10^{-10} p_{\infty}$. White and grey dashed lines represent the mean separation and reattachment points respectively.

$15^{\circ}$ respectively. Approximately an order of magnitude difference is obtained between each case as the angle of attack is increased. This is fairly consistent with the noise observed in the far-field at $\theta=90^{\circ}$ (see figure 
$7(a))$.

The same analysis can be carried out for the $\theta=0^{\circ}$ and $180^{\circ}$ observer locations in order to determine the cause for the different radiated noise trends shown at these locations in the far-field (figure 7 (c) and $(d))$. Figure 10 shows contours of $L_{w}$ at two Strouhal numbers, $S t=0.75$ and $S t=23.00$, which correspond to the low frequency peak for $\alpha=15^{\circ}$, and the maximum high frequency increase for $\alpha=10^{\circ}$ relative to $15^{\circ}$. At $S t=0.75$, the $\alpha=10^{\circ}$ and $15^{\circ}$ cases dominate over the $\alpha=5^{\circ}$ case due to high strength LE and TE regions, which also remain relatively in-phase. For chord-wise observer directions the LE region tends to dominate if there are pressure fluctuations occurring there because a significant component of the local normal vector is in the radiation direction, which increases $L_{w}$ through the $\cos (\Phi)$ term. For this reason, the location of the separation bubble and also transition to turbulence near the LE for $\alpha=10^{\circ}$ may explain how there is a larger increase in high frequency noise for chord-wise directions relative to $\alpha=15^{\circ}$. This is supported by the $\left|L_{w}\right|$ contour plot for $S t=23.00$ which shows the highest value for $\alpha=10^{\circ}$ near the LE.

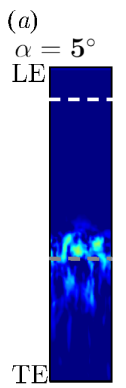

$\mathrm{St}=0.75$
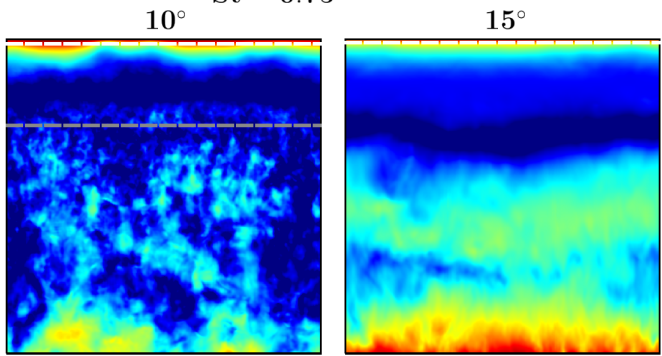

$20 \log _{10}\left(\left|L_{w}\right| / p_{\text {ref }}\right):{ }_{70}$
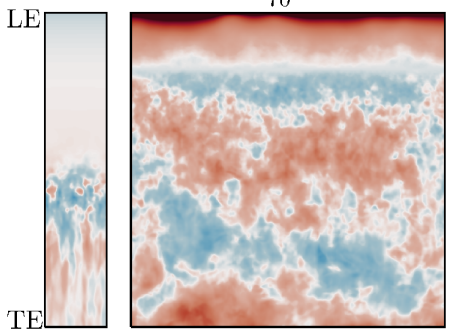

$L_{w} \mid \sin (\phi) / p_{\infty}:$
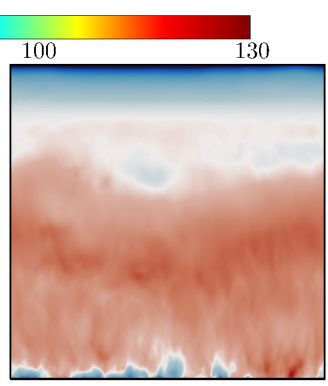

0 (b)

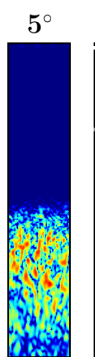

$\mathrm{St}=23.00$
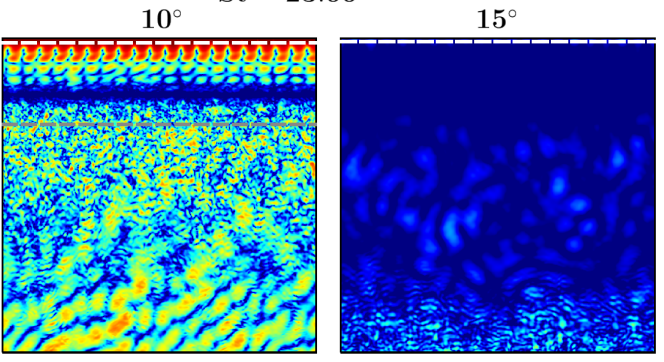
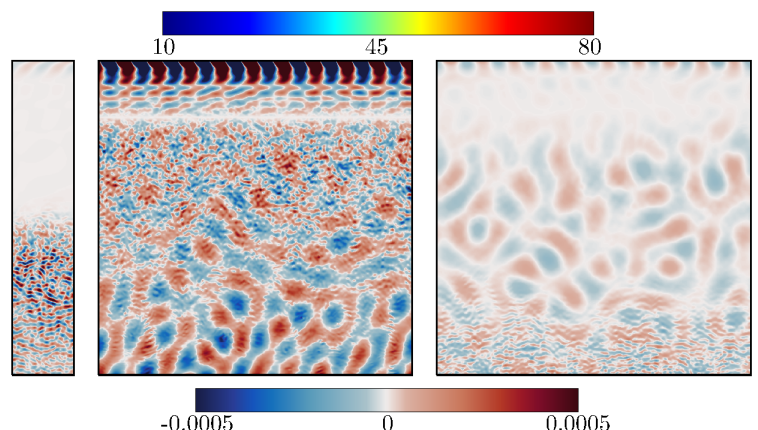

Figure 10: Fourier transform of $l_{w}$ (see 11) for the three angles of attack for an observer at $\left(x_{o}, y_{o}, z_{o}\right)=\left(-10 L_{c}, 0,0\right)$. Two frequencies are shown: $S t=0.75(a), S t=23.00(b)$. The upper row shows Fourier transform magnitude (in logarithmic scale), while the lower row shows the imaginary part of Fourier transform. The reference pressure used is $p_{\text {ref }}=10^{-10} p_{\infty}$. White and grey dashed lines represent the mean separation and reattachment points respectively.

\subsection{Noise source mechanisms}

In this section further discussion of the flow structures responsible for the high intensity source areas shown in the previous section are made. We consider a frequency filtered solution of the fluctuating pressure field through the following procedure: Firstly, the Fourier transform in time is taken at every point in the grid. Then, we multiply by a narrow band weighting function centred at a desired target frequency. Next, the inverse Fourier transform is performed, thereby producing a filtered solution as a function of time and space. The weighting function is given as follows:

$$
\begin{gathered}
\widetilde{p}_{a}(\boldsymbol{x}, t)=\mathcal{F}^{-1}\left\{W(f) \mathcal{F}\left[p_{a}(\boldsymbol{x}, t)\right]\right\}, \\
W(f)=\exp \left[-(\beta)\left(f-f_{p}\right)^{2} / \Delta f^{2}\right]+\exp \left[-(\beta)\left(f+f_{p}\right)^{2} / \Delta f^{2}\right],
\end{gathered}
$$

where $W(f)$ is the narrow-band frequency filter with a bandwidth set to $\Delta f^{*}=1, \mathcal{F}$ and $\mathcal{F}^{-1}$ represent the Fourier and inverse Fourier transforms; and, $\beta=20$ is a weighting coefficient inversely proportional to 
the broadness of the frequency range to be considered. Since the Fourier transform is two-sided, the filter is applied to both positive and negative frequency components. Figure 11 shows the filtered pressure field for $\alpha=15^{\circ}$ at six instances of time in $\Delta t^{*}=0.8$ intervals for $t^{*}=195.2$ to 199.2 (where $t^{*}=t a_{\infty} / L_{c}$ ) for $S t_{p}=f_{p} L_{c} / u_{\infty}=0.75$. Figure 11( $(a)$ shows a zoomed out view revealing the radiated sound field, while figure 11(b) shows the fluctuating pressure near the aerofoil. At this frequency the radiated noise appears to be dipolar. The dominant flow structures are vortices generated in the separated shear layer and at the TE which pair up to create a von Kármán vortex street shed into the wake. In frame (i) a vortex is shed from the LE shear layer which we can track as it convects over the surface and past the TE at (iv). During this time it induces a strong pressure signature on the wall. Once the vortex reaches the TE a dipole pulse is emitted, seen clearly on the aerofoil pressure side by the expansion wave travelling upstream in frames (ii)-(vi). These results confirm the experimental observations made by Lacagnina et al. [8], who suggested that a possible stall noise mechanism could be convection and scattering of wall pressure at the TE generated by nearby shear layer instabilities. The shear layer vortices are strongest from around the mid-chord location to the TE. This therefore explains why a high strength area was observed over the whole aft portion of the aerofoil surface at this frequency in figure $8(a)$.
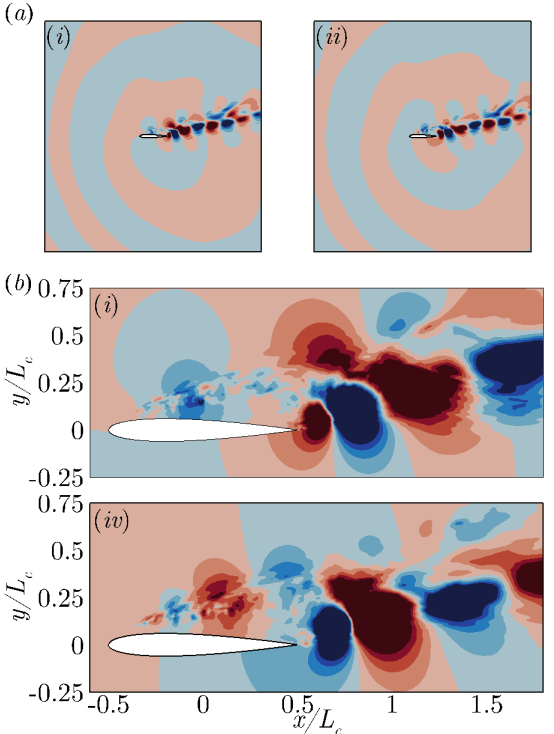
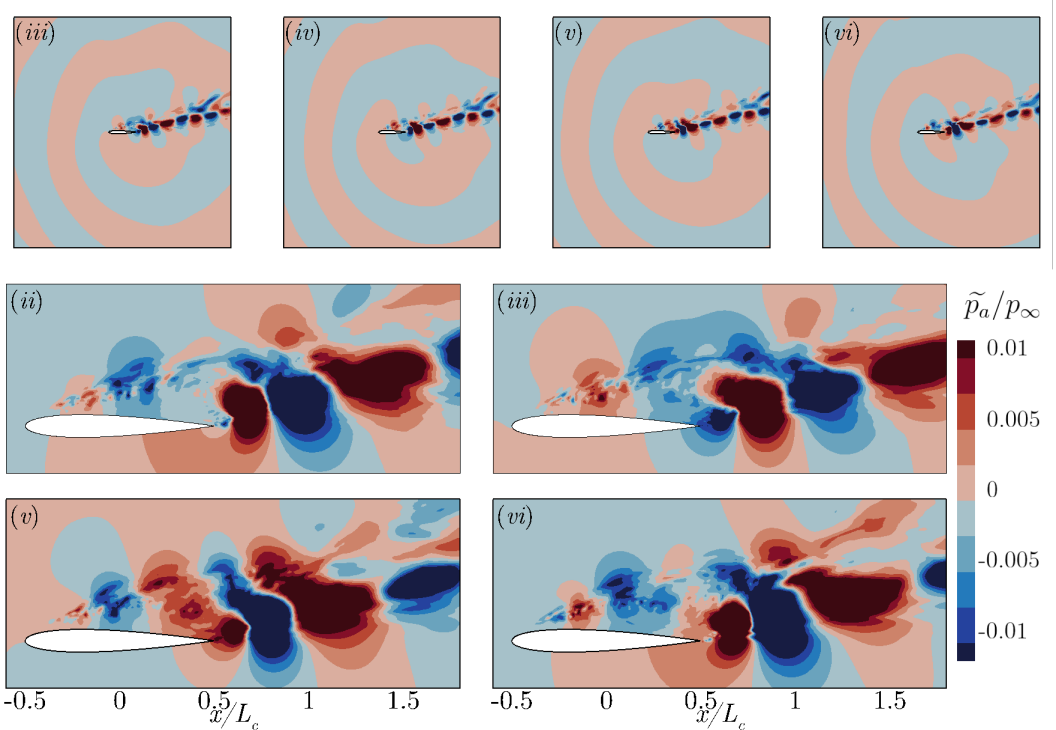

Figure 11: Snapshots of frequency-filtered pressure at $S t_{p}=0.75$ for the full-stall case $\alpha=15^{\circ}$. ( $a$ ) Zoomed out view, $(b)$ close up near aerofoil. Six instances of time are shown: (i)-(vi) which correspond to times $t^{*}=195.2$ to 199.2 in $\Delta t^{*}=0.8$ intervals.

The same analysis is carried out for a Strouhal number of $S t_{p}=3.00$ in figure 12 Similar to the lower frequency, the dominant flow structures appear in the LE shear layer and downstream from the TE. However, at this frequency the shear layer structures clearly have highest strength when they are located close to the wall, before approximately the mid-chord point. This explains why a spanwise strip of high source strength was observed for medium frequencies in the previous section close to the LE (see figure $8(b)$ and $9(a)$ ). The other high source strength area shown previously for $\alpha=15^{\circ}$ at this frequency was close to the TE. Due to the low strength of the shear layer vortices and their distance from the aerofoil surface when above the TE, it seems unlikely that the TE source region is determined through the same mechanism as at $S t=0.75$. Alternatively, the high strength TE source might be caused by scattering of the fluctuating pressure induced by the vortices shed at the TE and in the wake.

A comparison of the filtered pressure field for $\alpha=15^{\circ}$ is made with the other two angles of attack for three frequencies: $S t_{p}=0.75,3.00$, and 20.00 in figure $13(a)-(c)$. For all three frequencies the highest pressure fluctuations for $\alpha=5^{\circ}$ and $10^{\circ}$ appear close to the separation bubble reattachment point where transition takes place. This is consistent with the wall pressure data shown earlier. At the lowest frequency the highest strength flow structures are observed for the $\alpha=15^{\circ}$ case, also confirming the previous findings. 
(a)
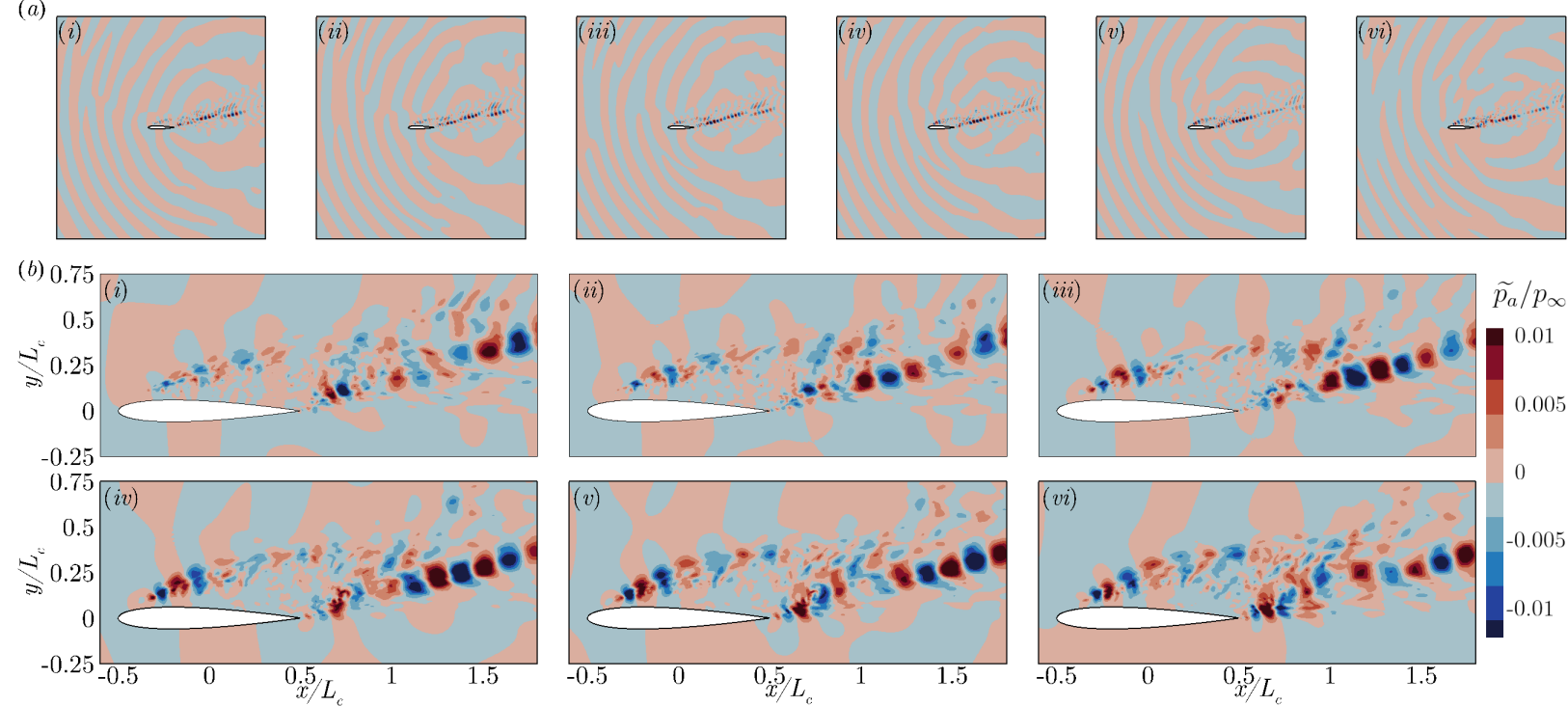

Figure 12: Snapshots of frequency-filtered pressure at $S t_{p}=3.00$ for the full-stall case $\alpha=15^{\circ}$. (a) Zoomed out view, $(b)$ close up near aerofoil. Six instances of time are shown: (i)-(vi) which correspond to times $t^{*}=195.2$ to 199.2 in $\Delta t^{*}=0.8$ intervals.

For the medium and high frequencies a similar maximum fluctuating pressure is obtained for both $\alpha=10^{\circ}$ and $15^{\circ}$. However, since the flow structures for the full-stall are de-attached from the surface the induced pressure on the wall is significantly lower. Despite this, the length of the highlighted flow structures appears to be slightly larger for the stall case at the low and medium frequencies which may explain why the phase changes less over the aerofoil surface. Similarly, at the high frequency it is apparent how the pressure fluctuations on the wall change sign more gradually when moving from LE to TE compared to the other two cases since the small scale high intensity flow structures are offset from the surface. At high frequencies there is clearly a dipole sound field generated for $\alpha=5^{\circ}$. The sound radiates mainly from the TE, but also to some extent from the bubble reattachment point as can be seen in figure $13(c)$. Similar observations have also been made previously for a NACA0006 aerofoil [29]. Comparatively, for $\alpha=10^{\circ}$ and $15^{\circ}$ there is evidence that other sources contribute significantly to the noise radiation due to the interference patterns present. This might indicate that at high frequencies direct quadrupole sources are also making a significant contribution to the radiated noise. Comparison of the dipole noise quantified in this paper with direct quadrupole sound will be the subject of a future work by the authors.

\section{Conclusions}

High order numerical simulations of a NACA0012 aerofoil at a low Reynolds number $R e_{\infty}=50,000$, and moderate Mach number $M=0.4$, are carried out in order to investigate the aerodynamic noise generated by flow separation and stall. Three flow conditions are considered: $\alpha=5^{\circ}$ where the aerofoil encounters a laminar separation bubble; $\alpha=10^{\circ}$ which is near stall; and, $\alpha=15^{\circ}$ full-stall condition. The study is based on a direct numerical simulation approach for the hydrodynamic flow field, with a time domain $\mathrm{FW}-\mathrm{H}$ solver used to predict the far-field sound. The focus of the work is the dipole noise contribution to the radiated sound. A number of significant findings are made concerning the radiated noise characteristics and noise generation mechanisms in stall:

(i) Three frequency ranges are identified for the far-field noise in the current flow configuration. Low frequency $S t \leq 1.5$ where the stall case dominates. Medium frequency $1.5<S t<10$ where a comparable level of noise is maintained for the three angles of attack. High frequency $S t \geq 10$ where the near stall case produces the highest dipole noise levels. 

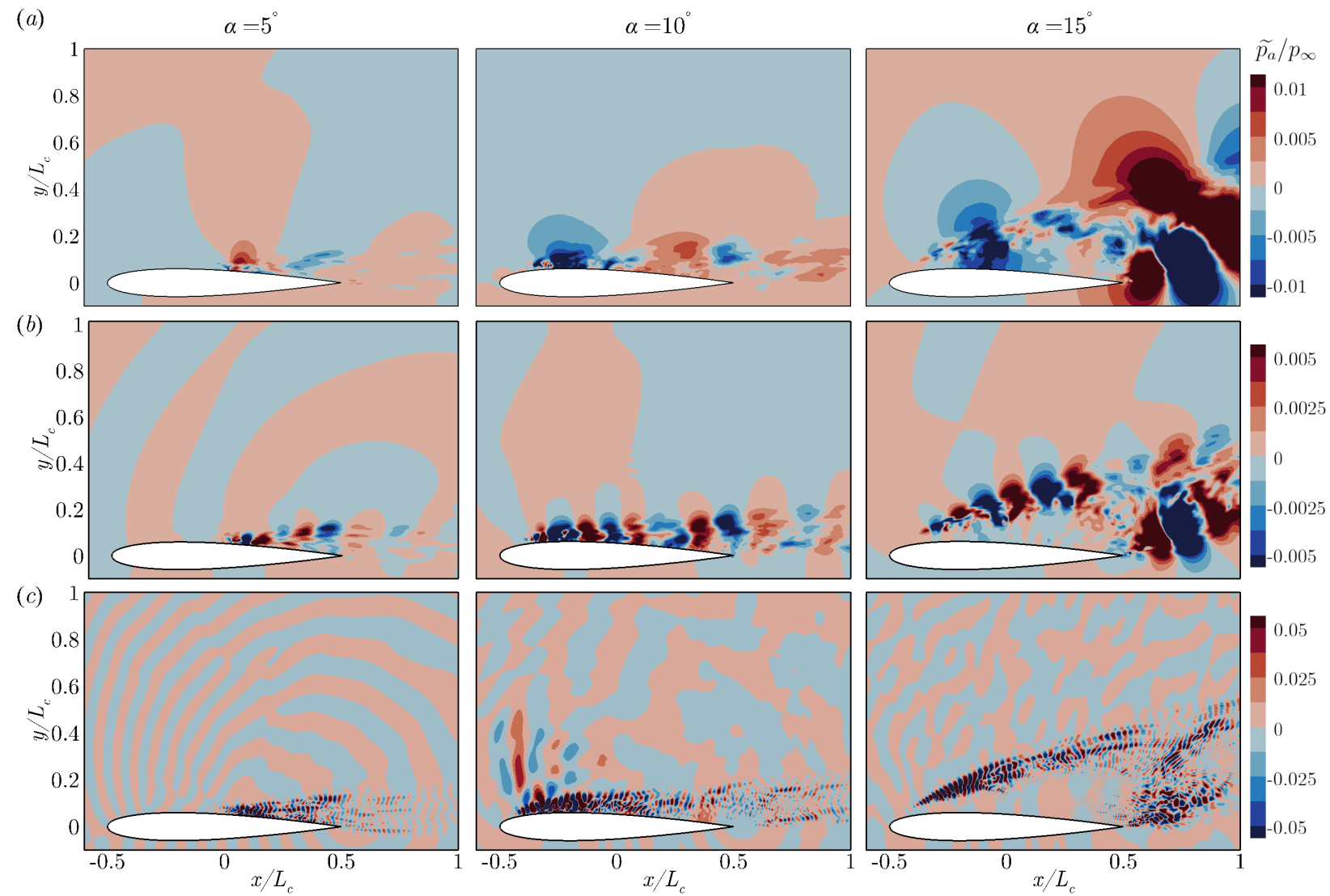

Figure 13: An instantaneous snapshot of the frequency-filtered pressure field solution (via [13p) taken at the end of each simulations. (a) $S t_{p}=0.75$, (b) $S t_{p}=3.00$, (c) $S t_{p}=20.00$.

(ii) These trends are fairly consistent for all observer angles. However, at higher frequencies the stall case tends to produce more directed sound than the other cases.

(iii) For vertical observer locations at low frequencies a high source amplitude is observed over the aft portion of the aerofoil when under stall conditions. This is revealed by examining the frequency and space distribution of the leading term in the FW-H equation over the whole aerofoil surface. Comparatively for the two lower angles of attack the strongest fluctuations are recorded at the separation bubble reattachment/transition regions.

(iv) At medium frequencies the stall case shows high strength source areas close to the TE and near the LE. The source amplitude is shown to be considerably weaker than the lower angles of attack, but is compensated by a more in-phase source relationship resulting in more efficient noise radiation.

(v) A similar trend is also observed for high frequencies, although the LE source is less prominent.

(vi) For low frequencies filtered snapshots of the pressure field show formation of shear layer vortices at around the mid-chord location. These induce strong pressure fluctuations on the wall as they convect towards the TE before being scattered as a dipole pulse. This identifies the cause of the high source strength on the aft portion of the aerofoil, and confirms one of the suggested stall noise mechanisms of Lacagnina et al. 8 .

(vii) At medium frequency vortices generated in the separated shear layer are responsible for the high intensity source near the LE. However these structures weaken considerably before reaching the TE. 
For this reason the TE sources may be caused by scattering of fluctuating pressure induced by the vortices shed at the TE and in the wake.

The prominence of high frequency dipole noise at $\alpha=10^{\circ}$ is caused by the proximity of high intensity structures to the wall, whereas in stall they are significantly offset due to appearing in the separated shear layer.

Currently the literature on aerofoil stall noise is underdeveloped and therefore there is a large parameter space to consider in order to identify the noise generation mechanisms in a general sense. In the current work the dipole noise mechanisms were investigated in detail for the current flow configuration which considers a LE stall scenario, where fully separated flow is developed by the generation and eventually bursting of a laminar separation bubble. It is nessecary for a future study to also consider higher Reynolds number flows in order to verify how the mechanisms might change when trailing edge stall is prominent. This however may require lower fidelity techniques in order to consider aerofoil simulations with large enough span lengths.

420 Additionally, a question remains regarding the influence of direct quadrupole noise in stall which warrants some additional study in the future.

\section{ACKNOWLEDGEMENT}

The authors gratefully acknowledge the support of the ESPRC (Engineering and Physical Sciences Research Council) under grant (EP/R010900/1). We also acknowledge the high-performance computing facilities and services offered by the UK national supercomputer ARCHER, and the local IRIDIS5 at the University of Southampton. All data supporting this study are openly available from the University of Southampton repository at http://dx.doi.org/10.5258/SOTON/XXXXXX.

\section{References}

[1] A. Laratro, M. Arjomandi, R. Kelso, B. Cazzolato, A discussion of wind turbine interaction and stall contributions to wind farm noise, J. Wind Eng. Ind. Aerodyn. 127 (2014) 1-10.

[2] M. R. Fink, D. A. Bailey, Airframe noise reduction studies and clean-airframe noise investigation, NASA CR-159311.

[3] R. W. Paterson, R. K. Amiet, C. L. Munch, Isolated airfoil-tip vortex interaction noise, J. Aircraft 12 (1) (1974) $34-40$.

[4] T. F. Brooks, D. S. Pope, M. A. Marcolini, Airfoil self-noise and prediction, NASA reference publication 1218.

[5] S. Moreau, M. Roger, J. Christophe, Flow features and self-noise of airfoils near stall or in stall, in: 15th AIAA/CEAS Aeroacoustics Conference. AIAA Paper 2009-3198, 2009.

[6] F. Bertagnolio, H. A. Madsen, A. Fischer, C. Bak, Experimental characterization of stall noise, in: 6th International Conference on Wind Turbine Noise, 2017.

[7] A. Laratro, M. Arjomandi, B. Cazzolato, R. Kelso, Self-noise of NACA0012 and NACA0021 aerofoil at the onset of stall, Int. J. Aeroacoust. 16 (3) (2017) 181-195.

[8] G. Lacagnina, P. Chaitanya, T. Berk, J. H. Kim, P. Joseph, B. Ganapathisbrumani, S. M. Hasheminejad, T. P. Chong, O. Stalnov, K. S. Choi, M. F. Shahab, M. Omidyeganeh, A. Pinelli, Mechanisms of airfoil noise near stall conditions, Phys. Rev. Fluids 4123902.

[9] Y. D. Mayer, B. Zang, M. Azarpeyvand, Aeroacoustic characteristics of a NACA 0012 airfoil for attached and stalled flow conditions, in: 25th AIAA/CEAS Aeroacoustics Conference. AIAA Paper 2019-2530, 2019.

10] J. W. Kim, P. J. Morris, Computation of subsonic inviscid flow past a cone using high-order schemes, AIAA J. 40 (10) (2002) 1961-1968.

[11] F. M. White, Viscous Fluid Flow, McGraw-Hill, 1991.

[12] J. W. Kim, D. J. Lee, Generalized characteristic boundary conditions for computational aeroacoustics, AIAA J. 38 (11) (2000) 2040-2049.

[13] J. W. Kim, A. S. H. Lau, N. D. Sandham, CAA boundary conditions for airfoil noise due to high-frequency gusts, Proc. Eng. 6 (2010) 244-253.

[14] J. W. Kim, A. S. H. Lau, N. D. Sandham, Proposed boundary conditions for gust-airfoil interaction noise, AIAA J. 48 (11) (2010) 2705-2709.

[15] J. W. Kim, D. J. Lee, Generalized characteristic boundary conditions for computational aeroacoustics, part 2, AIAA J. 42 (1) (2004) 47-55.

[16] J. W. Kim, Optimised boundary compact finite difference schemes for computational aeroacoustics, J. Comput. Phys. 225 (2007) 995-1019.

[17] J. W. Kim, High-order compact filters with variable cut-off wavenumber and stable boundary treatment, Comput. Fluids 39 (2010) 1168-1182. 
[18] J. W. Kim, Quasi-disjoint pentadiagonal matrix systems for the parallelization of compact finite-difference schemes and filters, J. Comput. Phys. 241 (2013) 168-194.

[19] J. M. Turner, J. W. Kim, Effect of spanwise domain size on direct numerical simulations of airfoil noise during flow separation and stall, under review in Phys. Fluids.

[20] N. J. Georgiadis, D. P. Rizzetta, C. Fureby, Large-eddy simulations: Current capabilities recommended practices, and future research, AIAA J. 48 (8) (2010) 1772-1784.

[21] S. Laizet, J. Nedić, C. Vassilicos, Influence of the spatial resolution on fine-scale features in DNS of turbulence generated by a single square grid, Int. J. Comput. Fluid D. 29 (3-5) (2015) 286-302.

[22] J. E. Ffowcs Williams, D. Hawkings, Sound generation by turbulence and surface in arbitrary motion, Philos. Trans. R. Soc. Lond. A 264 (1969) 321-342.

470 [23] F. Farassat, Derivations of formulations 1 and 1a of Farassat, NASA/TM-2007-214853.

[24] I. E. Garrick, C. E. Watkins, A theoretical study of the effect of forward speed on the free-space sound-pressure field around propellers, NACA TN-3018.

[25] J. M. Turner, Aerodynamic noise from undulated leading edge aerofoils, PhD Thesis.

[26] M. E. Goldstein, Aeroacoustics, McGraw-Hill, 1976.

475 [27] L. E. Jones, R. D. Sandberg, N. D. Sandham, Direct numerical simulations of forced and unforced separation bubbles on an airfoil at incidence, J. Fluid Mech. 602 (2008) 175-207.

[28] W. R. Wolf, J. L. F. Azevedo, S. K. Lele, Convective effects and the role of quadrupole sources for aerofoil aeroacoustics, J. Fluid Mech. 708 (2012) 502-538.

[29] R. D. Sandberg, L. E. Jones, Direct numerical simulations of airfoil self-noise, Proc. Eng. 6 (2010) 274-282. 